\title{
A New Iterative Method for Finding Common Solutions of a System of Equilibrium Problems, Fixed-Point Problems, and Variational Inequalities
}

\author{
Jian-Wen Peng, ${ }^{1}$ Soon-Yi Wu, ${ }^{2}$ and Jen-Chih Yao ${ }^{3}$ \\ ${ }^{1}$ School of Mathematics, Chongqing Normal University, Chongqing 400047, China \\ ${ }^{2}$ Department of Mathematics, National Cheng Kung University, Tainan 701, Taiwan \\ ${ }^{3}$ Department of Applied Mathematics, National Sun Yat-sen University, Kaohsiung 804, Taiwan
}

Correspondence should be addressed to Jian-Wen Peng, jwpeng6@yahoo.com.cn

Received 18 February 2010; Revised 22 May 2010; Accepted 22 June 2010

Academic Editor: Simeon Reich

Copyright (c) 2010 Jian-Wen Peng et al. This is an open access article distributed under the Creative Commons Attribution License, which permits unrestricted use, distribution, and reproduction in any medium, provided the original work is properly cited.

We introduce a new iterative scheme based on extragradient method and viscosity approximation method for finding a common element of the solutions set of a system of equilibrium problems, fixed point sets of an infinite family of nonexpansive mappings, and the solution set of a variational inequality for a relaxed cocoercive mapping in a Hilbert space. We prove strong convergence theorem. The results in this paper unify and generalize some well-known results in the literature.

\section{Introduction}

Let $H$ be a real Hilbert space with inner product $\langle\cdot, \cdot\rangle$ and induced norm $\|\cdot\|$. Let $C$ be a nonempty, closed, and convex subset of $H$. Let $\left\{F_{k}\right\}_{k \in \Gamma}$ be a countable family of bifunctions from $C \times C$ to $\mathbb{R}$, where $\mathbb{R}$ is the set of real numbers. Combettes and Hirstoaga [1] considered the following system of equilibrium problems:

Find $x \in C$ such that $(\forall k \in \Gamma),(\forall y \in C), F_{k}(x, y) \geq 0$.

If $\Gamma$ is a singleton, problem (1.1) becomes the following equilibrium problem:

Finding $x \in C$ such that $F(x, y) \geq 0, \quad \forall y \in C$. 
The solutions set of (1.2) is denoted by $\operatorname{EP}(F)$. And clearly the solutions set of problem (1.1) can be written as $\bigcap_{k \in \Gamma} \mathrm{EP}\left(F_{k}\right)$.

Problem (1.1) is very general in the sense that it includes, as special cases, optimization problems, variational inequalities, minimax problems, Nash equilibrium problem in noncooperative games, and others; see for instance, [1-4].

Recall that a mapping $S$ of a closed and convex subset $C$ into itself is nonexpansive if

$$
\|S x-S y\| \leq\|x-y\| \quad \forall x, y \in C .
$$

We denote fixed-points set of $S$ by $\operatorname{Fix}(S)$. A mapping $f: C \rightarrow C$ is called contraction if there exists a constant $\alpha \in(0,1)$ such that

$$
\|f x-f y\| \leq \alpha\|x-y\|, \quad \forall x, y \in C .
$$

A bounded linear operator $B$ on $H$ is strongly positive, if there is a constant $\bar{\gamma}>0$ such that $\langle B x, x\rangle \geq \bar{\gamma}\|x\|^{2}$ for all $x \in H$.

Combettes and Hirstoaga [1] introduced an iterative scheme for finding a common element of the solutions set of problem (1.1) in a Hilbert space and obtained a weak convergence theorem. Peng and Yao [2] introduced a new viscosity approximation scheme based on the extragradient method for finding a common element in the solutions set of the problem (1.1), fixed-points set of an infinite family of nonexpansive mappings and the solutions set of the variational inequality for a monotone and Lipschitz continuous mapping in a Hilbert space and obtained a strong convergence theorem. Colao et al. [3] introduced an implicit method for finding common solutions of variational inequalities and systems of equilibrium problems and fixed-points of infinite family of nonexpansive mappings in a Hilbert space and obtained a strong convergence theorem. Saeidi [4] introduced some iterative algorithms for finding a common element of the solutions set of a system of equilibrium problems and of fixed-points set of a finite family and a left amenable semigroup of nonexpansive mappings in a Hilbert space and obtained some strong convergence theorems.

Several algorithms for problem (1.2) have been proposed (see [5-20]). S. Takahashi and W. Takahashi [5] introduced and studied the following iterative scheme by the viscosity approximation method for finding a common element of the solutions set of problem (1.2) and fixed-points set of a nonexpansive mapping in a Hilbert space. Let an arbitrary $x_{1} \in H$ define sequences $\left\{x_{n}\right\}$ and $\left\{u_{n}\right\}$ by

$$
\begin{gathered}
F\left(u_{n}, y\right)+\frac{1}{\beta_{n}}\left\langle y-u_{n}, u_{n}-x_{n}\right\rangle \geq 0, \quad \forall y \in C, \\
x_{n+1}=\alpha_{n} f\left(x_{n}\right)+\left(1-\alpha_{n}\right) S u_{n}, \quad \forall n \in N .
\end{gathered}
$$

Shang et al. [6] introduced the following iterative scheme by the viscosity approximation method for finding a common element of the solutions set of problem (1.2) and fixed-points 
set of a nonexpansive mapping in a Hilbert space. Let an arbitrary $x_{1} \in H$, define sequences $\left\{x_{n}\right\}$ and $\left\{u_{n}\right\}$ by

$$
\begin{gathered}
F\left(u_{n}, y\right)+\frac{1}{\beta_{n}}\left\langle y-u_{n}, u_{n}-x_{n}\right\rangle \geq 0, \quad \forall y \in C \\
x_{n+1}=\alpha_{n} \gamma f\left(x_{n}\right)+\left(I-\alpha_{n} B\right) S u_{n}, \quad \forall n \in N
\end{gathered}
$$

They proved that under certain appropriate conditions imposed on $\left\{\alpha_{n}\right\}$ and $\left\{\beta_{n}\right\}$, the sequences $\left\{x_{n}\right\}$ and $\left\{u_{n}\right\}$ generated by (1.6) converge strongly to the unique solution of the variational inequality

$$
\left\langle(B-\gamma f) x^{*}, x-x^{*}\right\rangle \geq 0, \quad \forall x \in \operatorname{Fix}(S) \cap \operatorname{EP}(F)
$$

which is the optimality condition for the minimization problem

$$
\min _{x \in \operatorname{Fix}(S) \cap \operatorname{EP}(F)} \frac{1}{2}\langle B x, x\rangle-h(x)
$$

where $h$ is a potential function for $\gamma f$ (i.e., $h^{\prime}(x)=\gamma f(x)$ for $x \in H$ ). If $C=H$, the algorithm (1.6) was also studied by Plubtieng and Punpaeng [7].

Let $A: C \rightarrow H$ be a monotone mapping. The variational inequality problem is to find a point $x \in C$ such that

$$
\langle A x, y-x\rangle \geq 0
$$

for all $y \in C$. The solutions set of the variational inequality problem is denoted by $\operatorname{VI}(C, A)$. Qin et al. [8] introduced the following general iterative scheme for finding a common element of the solutions set of problem (1.2), the solutions set of a variational inequality and fixedpoints set of a nonexpansive mapping in a Hilbert space. Let an arbitrary $x_{1} \in H$, define sequences $\left\{x_{n}\right\}$ and $\left\{u_{n}\right\}$ by

$$
\begin{gathered}
F\left(u_{n}, y\right)+\frac{1}{\beta_{n}}\left\langle y-u_{n}, u_{n}-x_{n}\right\rangle \geq 0, \quad \forall y \in C, \\
x_{n+1}=\alpha_{n} \gamma f\left(x_{n}\right)+\left(I-\alpha_{n} B\right) S P_{C}\left(I-s_{n} A\right) u_{n}, \quad \forall n \in N .
\end{gathered}
$$

They proved that under certain appropriate conditions imposed on $\left\{\alpha_{n}\right\},\left\{s_{n}\right\}$ and $\left\{\beta_{n}\right\}$, the sequences $\left\{x_{n}\right\}$ and $\left\{u_{n}\right\}$ generated by (1.10) converge strongly to the unique solution of the variational inequality

$$
\left\langle(B-\gamma f) x^{*}, x-x^{*}\right\rangle \geq 0, \quad \forall x \in \operatorname{Fix}(S) \cap \operatorname{VI}(C, A) \cap \operatorname{EP}(F) .
$$

Qin et al. [9] introduced the following general iterative scheme for finding a common element of the solutions set of problem (1.2) and fixed-points set of a finite family of 
nonexpansive mappings in a Hilbert space. Let an arbitrary $x_{1} \in H$, define sequences $\left\{x_{n}\right\}$ and $\left\{u_{n}\right\}$ by

$$
\begin{gathered}
F\left(u_{n}, y\right)+\frac{1}{\beta_{n}}\left\langle y-u_{n}, u_{n}-x_{n}\right\rangle \geq 0, \quad \forall y \in C, \\
x_{n+1}=\alpha_{n} \gamma f\left(W_{n} x_{n}\right)+\left(1-\alpha_{n} B\right) W_{n} P_{C}\left(I-s_{n} A\right) u_{n}, \quad \forall n \in N,
\end{gathered}
$$

where $W_{n}$ is the $W$-mapping generated by $T_{1}, T_{2}, \ldots, T_{N}$ and $\lambda_{n 1}, \lambda_{n 2}, \ldots, \lambda_{n N}$. They proved that under certain appropriate conditions imposed on $\left\{\alpha_{n}\right\},\left\{s_{n}\right\}$ and $\left\{\beta_{n}\right\}$, the sequences $\left\{x_{n}\right\}$ and $\left\{u_{n}\right\}$ generated by (1.12) converge strongly to the unique solution of the variational inequality

$$
\left\langle(B-\gamma f) x^{*}, x-x^{*}\right\rangle \geq 0, \quad \forall x \in \bigcap_{i=1}^{N} \operatorname{Fix}\left(T_{i}\right) \cap \operatorname{VI}(C, A) \cap \operatorname{EP}(F)
$$

A typical problem is to minimize a quadratic function over the fixed-points set of a nonexpansive mapping $S$ on a real Hilbert space $H$, that is,

$$
\min _{x \in \operatorname{Fix}(S)} \frac{1}{2}\langle B x, x\rangle-\langle x, b\rangle,
$$

where $b$ is a given point in $H$. In 2003, $\mathrm{Xu}$ [21] proved that the sequence $\left\{x_{n}\right\}$ defined by the iterative method below, with the initial point $x_{0} \in H$, chosen arbitrarily:

$$
x_{n+1}=\left(I-\alpha_{n} B\right) S x_{n}+\alpha_{n} u, \quad n \geq 0,
$$

converges strongly to the unique solution of the minimization problem (1.15) provided the sequence $\left\{\alpha_{n}\right\}$ satisfies certain conditions. Marino and $\mathrm{Xu}$ [22] combine the iterative method (1.15) with the viscosity approximation in [23] and consider the following general iterative method: with the initial point $x_{0} \in H$, chosen arbitrarily:

$$
x_{n+1}=\left(1-\alpha_{n} B\right) S x_{n}+\alpha_{n} \gamma f\left(x_{n}\right), \quad n \geq 0 .
$$

They proved that if the sequence $\left\{\alpha_{n}\right\}$ satisfies appropriate conditions, then the sequence $\left\{x_{n}\right\}$ generated by (1.16) converges strongly to the unique solution of the variational inequality

$$
\left\langle(B-\gamma f) x^{*}, x-x^{*}\right\rangle \geq 0, \quad x \in \operatorname{Fix}(S)
$$

which is the optimality condition for the minimization problem

$$
\min _{x \in \operatorname{Fix}(S)} \frac{1}{2}\langle B x, x\rangle-h(x)
$$

where $h$ is a potential function for $\gamma f$. 
Recently, Qin et al. [24] introduced the following general iterative process: with the initial point $x_{1} \in C$, chosen arbitrarily:

$$
\begin{gathered}
y_{n}=P_{C}\left(I-s_{n} A\right) x_{n}, \\
x_{n+1}=\alpha_{n} \gamma f\left(W_{n} x_{n}\right)+\left(I-\alpha_{n} B\right) W_{n} P_{C}\left(I-r_{n} A\right) y_{n}, \quad \forall n \in N,
\end{gathered}
$$

where $W_{n}$ is the $W$-mapping generated by $T_{1}, T_{2}, \ldots, T_{N}$ and $\lambda_{n 1}, \lambda_{n 2}, \ldots, \lambda_{n N}$. They proved that if the sequences of parameters $\left\{\alpha_{n}\right\},\left\{r_{n}\right\}$ and $\left\{s_{n}\right\}$ satisfies appropriate conditions, then the sequence $\left\{x_{n}\right\},\left\{y_{n}\right\}$ generated by (1.19) converge strongly to a point $x^{*}$ which is the unique solution of the variational inequality

$$
\left\langle(B-\gamma f) x^{*}, x-x^{*}\right\rangle \geq 0, \quad \forall x \in \bigcap_{i=1}^{N} F\left(T_{i}\right) \cap \operatorname{VI}(C, A) .
$$

Inspired and motivated by above works, we introduce a new iterative scheme based on extragradient method and viscosity approximation method for finding a common element of the solutions set of a system of equilibrium problems, fixed-points set of a family of infinitely nonexpansive mappings and the solutions set of a variational inequality for a relaxed cocoercive mapping in a Hilbert space. We prove strong convergence theorem. The results in this paper unify, generalize and extend some well-known results in $[6-9,21,22,24]$.

\section{Preliminaries}

Let $H$ be a real Hilbert space with inner product $\langle\cdot, \cdot\rangle$ and norm $\|\cdot\|$. Let $C$ be a nonempty, closed, and convex subset of $H$. Let symbols $\rightarrow$ and $\rightarrow$ denote strong and weak convergence, respectively. It is well known that

$$
\|\lambda x+(1-\lambda) y\|^{2}=\lambda\|x\|^{2}+(1-\lambda)\|y\|^{2}-\lambda(1-\lambda)\|x-y\|^{2}
$$

for all $x, y \in H$ and $\lambda \in[0,1]$.

For any $x \in H$, there exists a unique nearest point in $C$, denoted by $P_{C}(x)$, such that $\left\|x-P_{C}(x)\right\| \leq\|x-y\|$ for all $y \in C$. The mapping $P_{C}$ is called the metric projection of $H$ onto $C$. We know that $P_{C}$ is a nonexpansive mapping from $H$ onto $C, P_{C}(x) \in C$ and

$$
\left\langle x-P_{C}(x), P_{C}(x)-y\right\rangle \geq 0
$$

for all $x, y \in H$.

It is easy to see that (2.2) is equivalent to

$$
\|x-y\|^{2} \geq\left\|x-P_{C}(x)\right\|^{2}+\left\|y-P_{C}(x)\right\|^{2}
$$

for all $x, y \in H$. It is also known that $P_{C}$ has the following firmly nonexpansive property:

$$
\left\langle x-y, P_{C} x-P_{C} y\right\rangle \geq\left\|P_{C} x-P_{C} y\right\|^{2}
$$

for all $x, y \in H$. 
Recall also that a mapping $A$ of $C$ into $H$ is called monotone if

$$
\langle A x-A y, x-y\rangle \geq 0
$$

for all $x, y \in C$. $A$ is said to be $\mu$-cocoercive, if for each $x, y \in C$, we have

$$
\langle A x-A y, x-y\rangle \geq \mu\|A x-A y\|^{2},
$$

for a constant $\mu>0$. A is said to be relaxed $(u, v)$-cocoercive, if there exist two constants $u, v>0$ such that

$$
\langle A x-A y, x-y\rangle \geq(-u)\|A x-A y\|^{2}+v\|x-y\|^{2}, \quad \forall x, y \in C
$$

Let $A$ be a monotone mapping of $C$ into $H$. In the context of the variational inequality problem the characterization of projection (2.2) implies the following:

$$
\begin{gathered}
u \in \operatorname{VI}(C, A) \Longrightarrow u=P_{C}(u-\lambda A u), \quad \lambda>0, \\
u=P_{C}(u-\lambda A u) \quad \text { for some } \lambda>0 \Longrightarrow u \in \operatorname{VI}(C, A) .
\end{gathered}
$$

It is also known that $H$ satisfies the Opial's condition [25], that is, for any sequence $\left\{x_{n}\right\} \subset H$ with $x_{n} \rightarrow x$, the inequality

$$
\liminf _{n \rightarrow \infty}\left\|x_{n}-x\right\|<\liminf _{n \rightarrow \infty}\left\|x_{n}-y\right\|
$$

holds for every $y \in H$ with $x \neq y$.

A set-valued mapping $T: H \rightarrow 2^{H}$ is called monotone if for all $x, y \in H, f \in T x$ and $g \in T y$ imply $\langle x-y, f-g\rangle \geq 0$. A monotone mapping $T: H \rightarrow 2^{H}$ is maximal if its graph $G(T)$ of $T$ is not properly contained in the graph of any other monotone mapping. It is known that a monotone mapping $T$ is maximal if and only if for $(x, f) \in H \times H,\langle x-y, f-g\rangle \geq 0$ for every $(y, g) \in G(T)$ implies $f \in T x$. Let $A$ be a monotone and $k$-Lipschitz-continuous mapping of $C$ into $H$ and let $N_{C} v$ be normal cone to $C$ at $v \in C$, that is, $N_{C} v=\{w \in H$ : $\langle v-u, w\rangle \geq 0$, for all $u \in C\}$. Define

$$
T v= \begin{cases}A v+N_{C} v & \text { if } v \in C \\ \emptyset & \text { if } v \notin C .\end{cases}
$$

Then $T$ is maximal monotone and $0 \in T v$ if and only if $v \in \operatorname{VI}(C, A)$ (see [26]).

For solving the problem (1.1), let us assume that the bifunction $F$ satisfies the following condition:

(A1) $F(x, x)=0$ for all $x \in C$;

(A2) $F$ is monotone, that is, $F(x, y)+F(y, x) \leq 0$ for any $x, y \in C$; 
(A3) for each $x, y, z \in C$,

$$
\lim _{t \downarrow 0} F(t z+(1-t) x, y) \leq F(x, y)
$$

(A4) for each $x \in C, y \mapsto F(x, y)$ is convex;

(A5) for each $x \in C, y \mapsto F(x, y)$ is lower semicontinuous.

We recall some lemmas needed later.

Lemma 2.1 (see $[1,10]$ ). Let $C$ be a nonempty, closed, and convex subset of $H$, and let $F$ be a bifunction from $C \times C$ to $\mathbb{R}$ which satisfies conditions (A1)-(A5). For $\beta>0$ and $x \in H$, define the mapping $T_{\beta}^{F}: H \rightarrow C$ as follows:

$$
T_{\beta}^{F}(x)=\left\{z \in C: F(z, y)+\frac{1}{\beta}\langle y-z, z-x\rangle \geq 0, \forall y \in C\right\}
$$

for all $x \in H$. Then, the following statements hold:

(1) $T_{\beta}^{F}(x) \neq \emptyset$;

(2) $T_{\beta}^{F}$ is single-valued;

(3) $T_{\beta}^{F}$ is firmly nonexpansive, that is, for any $x, y \in H$,

$$
\left\|T_{\beta}^{F}(x)-T_{\beta}^{F}(y)\right\|^{2} \leq\left\langle T_{\beta}^{F}(x)-T_{\beta}^{F}(y), x-y\right\rangle ;
$$

(4) $\operatorname{Fix}\left(T_{\beta}^{F}\right)=\mathrm{EP}(F)$;

(5) $\mathrm{EP}(F)$ is closed and convex.

Lemma 2.2 (see [27]). Assume that $\left\{s_{n}\right\}$ is a sequence of nonnegative real numbers such that

$$
s_{n+1} \leq\left(1-\alpha_{n}\right) s_{n}+\alpha_{n} \beta_{n}+\delta_{n}, \quad n \geq 1,
$$

where $\left\{\alpha_{n}\right\},\left\{\beta_{n}\right\}$ and $\left\{\delta_{n}\right\}$ are sequences of numbers which satisfy the conditions:

(i) $\left\{\alpha_{n}\right\} \subset[0,1], \sum_{n=1}^{\infty} \alpha_{n}=\infty$, or equivalently, $\prod_{i=1}^{\infty}\left(1-\alpha_{n}\right)=0$;

(ii) $\lim \sup _{n \rightarrow \infty} \beta_{n} \leq 0$;

(iii) $\delta_{n} \geq 0(n \geq 1), \sum_{n=1}^{\infty} \delta_{n}<\infty$;

Then, $\lim _{n \rightarrow \infty} s_{n}=0$.

Lemma 2.3. In a real Hilbert space $H$, the following inequality holds:

$$
\|x+y\|^{2} \leq\|x\|^{2}+2\langle y, x+y\rangle
$$

for all $x, y \in H$. 
Lemma 2.4 (see [22]). Assume that $A$ is a strongly positive linear bounded operator on a Hilbert space $H$ with coefficient $\tilde{\gamma}>0$ and $0<\rho \leq\|A\|^{-1}$. Then $\|I-\rho A\| \leq 1-\rho \tilde{\gamma}$.

Let $S_{1}, S_{2}, \ldots$ be a family of infinitely nonexpansive mappings of $C$ into itself and let $\xi_{1}, \xi_{2}, \ldots$ be real numbers such that $0 \leq \xi_{i} \leq 1$ for every $i \in N$. For any $n \in N$, define a mapping $W_{n}$ of $C$ into $C$ as follows:

$$
\begin{gathered}
U_{n, n+1}=I, \\
U_{n, n}=\xi_{n} S_{n} U_{n, n+1}+\left(1-\xi_{n}\right) I, \\
U_{n, n-1}=\xi_{n-1} S_{n-1} U_{n, n}+\left(1-\xi_{n-1}\right) I, \\
\vdots \\
U_{n, k}=\xi_{k} S_{k} U_{n, k+1}+\left(1-\xi_{k}\right) I, \\
U_{n, k-1}=\xi_{k-1} S_{k-1} U_{n, k}+\left(1-\xi_{k-1}\right) I, \\
\vdots \\
U_{n, 2}=\xi_{2} S_{2} U_{n, 3}+\left(1-\xi_{2}\right) I, \\
W_{n}=U_{n, 1}=\xi_{1} S_{1} U_{n, 2}+\left(1-\xi_{1}\right) I .
\end{gathered}
$$

Such a mapping $W_{n}$ is called the $W$-mapping generated by $S_{n}, S_{n-1}, \ldots, S_{1}$ and $\xi_{n}, \xi_{n-1}, \ldots, \xi_{1}$; see $[28,29]$.

Lemma 2.5 (see [28]). Let $C$ be a nonempty, closed, and convex subset of a Banach space E. Let $S_{1}, S_{2}, \ldots$ be a family of infinitely nonexpansive mappings of $C$ into itself such that $\bigcap_{i=1}^{\infty} \operatorname{Fix}\left(S_{i}\right)$ is nonempty, and let $\xi_{1}, \xi_{2}, \ldots$ be real numbers such that $0<\xi_{i} \leq d<1$ for every $i \in N$. For any $n \in N$, let $W_{n}$ be the $W$-mapping of $C$ into itself generated by $S_{n}, S_{n-1}, \ldots, S_{1}$ and $\xi_{n}, \xi_{n-1}, \ldots, \xi_{1}$. Then $W_{n}$ is asymptotically regular and nonexpansive. Further, if $E$ is strict convex, then $F\left(W_{n}\right)=\bigcap_{i=1}^{n} \operatorname{Fix}\left(S_{i}\right)$.

Lemma 2.6 (see [29]). Let $C$ be a nonempty, closed, and convex subset of a strictly convex Banach space $E$. Let $S_{1}, S_{2}, \ldots$ be a family of infinitely nonexpansive mappings of $C$ into itself such that $\bigcap_{i=1}^{\infty} \operatorname{Fix}\left(S_{i}\right)$ is nonempty, and let $\xi_{1}, \xi_{2}, \ldots$ be real numbers such that $0<\xi_{i} \leq d<1$ for every $i \in N$. Then for every $x \in C$ and $k \in N$, the limit $\lim _{n \rightarrow \infty} U_{n, k} x$ exists.

Remark 2.7. Using Lemma 2.6, one can define mappings $U_{\infty, k}$ and $W$ of $C$ into itself as follows:

$$
U_{\infty, k} x=\lim _{n \rightarrow \infty} U_{n, k} x
$$

and $W x=\lim _{n \rightarrow \infty} W_{n} x=\lim _{n \rightarrow \infty} U_{n, 1} x$ for every $x \in C$. Such a mapping $W$ is called the $W$-mapping generated by $S_{1}, S_{2}, \ldots$ and $\xi_{1}, \xi_{2}, \ldots$ Since $W_{n}$ is nonexpansive, $W: C \rightarrow C$ is also nonexpansive. Indeed, observe that for each $x, y \in C$

$$
\|W x-W y\|=\lim _{n \rightarrow \infty}\left\|W_{n} x-W_{n} y\right\| \leq\|x-y\|
$$


If $\left\{x_{n}\right\}$ is a bounded sequence in $C$, then we have

$$
\lim _{n \rightarrow \infty}\left\|W x-W_{n} x\right\|=0
$$

Lemma 2.8 (see [29]). Let $C$ be a nonempty, closed and convex subset of a strictly convex Banach space $E$. Let $S_{1}, S_{2}, \ldots$ be an infinite family of nonexpansive mappings of $C$ into itself such that $\bigcap_{i=1}^{\infty} \operatorname{Fix}\left(S_{i}\right)$ is nonempty, and let $\xi_{1}, \xi_{2}, \ldots$ be real numbers such that $0<\xi_{i} \leq d<1$ for every $i \in N$. Then $\operatorname{Fix}(W)=\bigcap_{n=1}^{\infty} \operatorname{Fix}\left(S_{n}\right)$.

\section{Strong Convergence Theorem}

In this section, we prove strong convergence theorem which solve the problem of finding a common element of the solutions set of a system of equilibrium problems, fixed-points set of a family of infinitely nonexpansive mappings, and the solutions set of a variational inequality for a relaxed cocoercive mapping in Hilbert space.

Theorem 3.1. Let $C$ be a nonempty, closed, and convex subset of $H$. Let $F_{1}, F_{2}, \ldots, F_{m}$ be bifunctions from $C \times C$ to $\mathbb{R}$ which satisfies conditions (A1)-(A5). Let $A: C \rightarrow H$ be relaxed $(u, v)$-cocoercive and $\mu$-Lipschitz continuous and $B$ a strongly positive linear bounded operator on $H$ with coefficient $\tilde{\gamma}>0$. Assume that $0<\gamma<\tilde{\gamma} / \alpha$. Let $S_{1}, S_{2}, \ldots$ be a family of infinitely nonexpansive mappings of $C$ into itself such that $\Omega=\bigcap_{i=1}^{\infty} \operatorname{Fix}\left(S_{i}\right) \cap \operatorname{VI}(C, A) \cap \bigcap_{k=1}^{m} \operatorname{EP}\left(F_{k}\right) \neq \emptyset$ and let $\xi_{1}, \xi_{2}, \ldots$ be real numbers such that $0<\xi_{i} \leq \delta<1$ for every $i \in N$, and let $W_{n}$ be the $W$-mapping of $C$ into itself generated by $S_{n}, S_{n-1}, \ldots, S_{1}$ and $\xi_{n}, \xi_{n-1}, \ldots, \xi_{1}$. Let $f: C \rightarrow C$ be a contraction with coefficient $\alpha(0<\alpha<1)$ and $\left\{x_{n}\right\},\left\{u_{n}\right\}$, and $\left\{y_{n}\right\}$ be sequences generated by

$$
\begin{gathered}
x_{1}=x \in H, \\
u_{n}=T_{\beta_{n}}^{F_{m}} T_{\beta_{n}}^{F_{m-1}} \cdots T_{\beta_{n}}^{F_{2}} T_{\beta_{n}}^{F_{1}} x_{n}, \\
y_{n}=P_{C}\left(I-s_{n} A\right) u_{n} \\
x_{n+1}=\alpha_{n} \gamma f\left(W_{n} x_{n}\right)+\left(I-\alpha_{n} B\right) W_{n} P_{C}\left(I-r_{n} A\right) y_{n}
\end{gathered}
$$

for every $n=1,2, \ldots$, where $\left\{\alpha_{n}\right\},\left\{\beta_{n}\right\},\left\{r_{n}\right\}$, and $\left\{s_{n}\right\}$ are sequences of numbers which satisfy the conditions:

(C1) $\left\{\alpha_{n}\right\} \subset[0,1]$ with $\lim _{n \rightarrow \infty} \alpha_{n}=0, \sum_{n=1}^{\infty} \alpha_{n}=\infty$, and $\sum_{n=1}^{\infty}\left|\alpha_{n+1}-\alpha_{n}\right|<\infty$;

(C2) $\left\{r_{n}\right\} \subset[a, b]$ and $\left\{s_{n}\right\} \subset[a, b]$ for some $a, b$ with $0 \leq a \leq b \leq 2\left(v-u \mu^{2}\right) / \mu^{2}, \sum_{n=1}^{\infty} \mid r_{n+1}-$ $r_{n} \mid<\infty$, and $\sum_{n=1}^{\infty}\left|s_{n+1}-s_{n}\right|<\infty$;

(C3) $\liminf _{n \rightarrow \infty} \beta_{n}>0$ and $\sum_{n=1}^{\infty}\left|\beta_{n+1}-\beta_{n}\right|<\infty$.

Then, $\left\{x_{n}\right\},\left\{y_{n}\right\}$, and $\left\{u_{n}\right\}$ converge strongly to a point $q \in \Omega$ which solves the following variational inequality:

$$
\langle\gamma f q-B q, p-q\rangle \leq 0, \quad \forall p \in \Omega
$$

Equivalently, one has $q=P_{\Omega}(\gamma f+(I-B))(q)$. 
Proof. Since $\alpha_{n} \rightarrow 0$ from condition (C1), we may assume, with no loss of generality, that $\alpha_{n} \leq\|B\|^{-1}$ for all $n$. Lemma 2.4 implies $\left\|I-\alpha_{n} B\right\| \leq 1-\alpha_{n} \tilde{\gamma}$. Next, we will assume that $\|I-B\| \leq 1-\tilde{\gamma}$. Now, we show that the mappings $I-s_{n} A$ and $I-r_{n} A$ are nonexpansive. Indeed, from the relaxed $(u, v)$-cocoercivity and $\mu$-Lipschitz continuity of $A$ and condition (C2), we have

$$
\begin{aligned}
\left\|\left(I-s_{n} A\right) x-\left(I-s_{n} A\right) y\right\|^{2} & =\left\|(x-y)-s_{n}(A x-A y)\right\|^{2} \\
& =\|x-y\|^{2}-2 s_{n}\langle x-y, A x-A y\rangle+s_{n}^{2}\|A x-A y\|^{2} \\
& \leq\|x-y\|^{2}-2 s_{n}\left[-u\|A x-A y\|^{2}+v\|x-y\|^{2}\right]+s_{n}^{2}\|A x-A y\|^{2} \\
& \leq\|x-y\|^{2}+2 s_{n} \mu^{2} u\|x-y\|^{2}-2 s_{n} v\|x-y\|^{2}+\mu^{2} s_{n}^{2}\|x-y\|^{2} \\
& =\left(1+2 s_{n} \mu^{2} u-2 s_{n} v+\mu^{2} s_{n}^{2}\right)\|x-y\|^{2} \\
& \leq\|x-y\|^{2},
\end{aligned}
$$

which implies the mapping $I-s_{n} A$ is nonexpansive, so is $I-r_{n} A$.

For $k \in\{0,1,2, \ldots, m\}$, and for any positive integer number $n$, we define the operator $\Theta_{\beta_{n}}^{k}: H \rightarrow C$ as follows:

$$
\begin{gathered}
\Theta_{\beta_{n}}^{0} x=x, \\
\Theta_{\beta_{n}}^{k} x=T_{\beta_{n}}^{F_{k}} T_{\beta_{n}}^{F_{k-1}} \cdots T_{\beta_{n}}^{F_{2}} T_{\beta_{n}}^{F_{1}} x, \quad k=1,2, \ldots, m .
\end{gathered}
$$

Next, we show that the sequence $\left\{x_{n}\right\}$ is bounded. Let $p \in \Omega$. Then from Lemma 2.1(3), we know that for $k \in\{1,2, \ldots, m\}, T_{\beta_{n}}^{F_{k}}$ is nonexpansive and $p=T_{\beta_{n}}^{F_{k}} p$, and

$$
\left\|u_{n}-p\right\|=\left\|\Theta_{\beta_{n}}^{m} x_{n}-p\right\|=\left\|\Theta_{\beta_{n}}^{m} x_{n}-\Theta_{\beta_{n}}^{m} p\right\| \leq\left\|x_{n}-p\right\|
$$

for all $n=1,2, \ldots$ By $p=P_{C}\left(I-s_{n} A\right) p$ and (3.5), we have

$$
\begin{aligned}
\left\|y_{n}-p\right\| & =\left\|P_{C}\left(I-s_{n} A\right) u_{n}-P_{C}\left(I-s_{n} A\right) p\right\| \\
& \leq\left\|\left(I-s_{n} A\right) u_{n}-\left(I-s_{n} A\right) p\right\| \leq\left\|u_{n}-p\right\| \leq\left\|x_{n}-p\right\| .
\end{aligned}
$$

Since $x_{n+1}=\alpha_{n} \gamma f\left(W_{n} x_{n}\right)+\left(I-\alpha_{n} B\right) W_{n} P_{C}\left(I-r_{n} A\right) y_{n}$ and $p=W_{n} p$, we have

$$
\begin{aligned}
\left\|x_{n+1}-p\right\| & =\left\|\alpha_{n}\left(\gamma f\left(W_{n} x_{n}\right)-B p\right)+\left(I-\alpha_{n} B\right)\left(W_{n} P_{C}\left(I-r_{n} A\right) y_{n}-p\right)\right\| \\
& \leq \alpha_{n}\left\|\gamma f\left(W_{n} x_{n}\right)-B p\right\|+\left(1-\alpha_{n} \tilde{\gamma}\right)\left\|P_{C}\left(I-r_{n} A\right) y_{n}-p\right\| \\
& \leq \alpha_{n} \gamma\left\|f\left(W_{n} x_{n}\right)-f(p)\right\|+\alpha_{n}\|\gamma f(p)-B p\|+\left(1-\alpha_{n} \tilde{\gamma}\right)\left\|y_{n}-p\right\| \\
& \leq\left[1-\alpha_{n}(\tilde{\gamma}-\alpha \gamma)\right]\left\|x_{n}-p\right\|+\alpha_{n}\|\gamma f(p)-B p\| .
\end{aligned}
$$


By inductions, we have

$$
\left\|x_{n}-p\right\| \leq \max \left\{\left\|x_{0}-p\right\|, \frac{\|\gamma f(p)-B p\|}{\tilde{r}-\alpha \gamma}\right\}
$$

which proves that the sequence $\left\{x_{n}\right\}$ is bounded. It follows from (3.5) and (3.6) that $\left\{y_{n}\right\}$ and $\left\{u_{n}\right\}$ are also bounded.

Since $\Theta_{\beta_{n}}^{k} x_{n}=T_{\beta_{n}}^{F_{k}} \Theta_{\beta_{n}}^{k-1} x_{n}$ and $\Theta_{\beta_{n+1}}^{k} x_{n+1}=T_{\beta_{n+1}}^{F_{k}} \Theta_{\beta_{n+1}}^{k-1} x_{n+1}$ for each $k=1,2, \ldots, m$, by Lemma 2.1, we have

$$
\begin{gathered}
F_{k}\left(\Theta_{\beta_{n}}^{k} x_{n}, y\right)+\frac{1}{\beta_{n}}\left\langle y-\Theta_{\beta_{n}}^{k} x_{n}, \Theta_{\beta_{n}}^{k} x_{n}-\Theta_{\beta_{n}}^{k-1} x_{n}\right\rangle \geq 0 \quad \forall y \in C, \\
F_{k}\left(\Theta_{\beta_{n+1}}^{k} x_{n+1}, y\right)+\frac{1}{\beta_{n+1}}\left\langle y-\Theta_{\beta_{n+1}}^{k} x_{n+1}, \Theta_{\beta_{n+1}}^{k} x_{n+1}-\Theta_{\beta_{n+1}}^{k-1} x_{n+1}\right\rangle \geq 0 \quad \forall y \in C,
\end{gathered}
$$

Setting $y=\Theta_{\beta_{n+1}}^{k} x_{n+1}$ in (3.9) and $y=\Theta_{\beta_{n}}^{k} x_{n}$ in (3.10), we have

$$
\begin{gathered}
F_{k}\left(\Theta_{\beta_{n}}^{k} x_{n}, \Theta_{\beta_{n+1}}^{k} x_{n+1}\right)+\frac{1}{\beta_{n}}\left\langle\Theta_{\beta_{n+1}}^{k} x_{n+1}-\Theta_{\beta_{n}}^{k} x_{n}, \Theta_{\beta_{n}}^{k} x_{n}-\Theta_{\beta_{n}}^{k-1} x_{n}\right\rangle \geq 0, \\
F_{k}\left(\Theta_{\beta_{n+1}}^{k} x_{n+1}, \Theta_{\beta_{n}}^{k} x_{n}\right)+\frac{1}{\beta_{n+1}}\left\langle\Theta_{\beta_{n}}^{k} x_{n}-\Theta_{\beta_{n+1}}^{k} x_{n+1}, \Theta_{\beta_{n+1}}^{k} x_{n+1}-\Theta_{\beta_{n+1}}^{k-1} x_{n+1}\right\rangle \geq 0 .
\end{gathered}
$$

Adding the two inequalities and from the monotonicity of $F$, we get

$$
\left\langle\Theta_{\beta_{n+1}}^{k} x_{n+1}-\Theta_{\beta_{n}}^{k} x_{n}, \frac{\Theta_{\beta_{n}}^{k} x_{n}-\Theta_{\beta_{n}}^{k-1} x_{n}}{\beta_{n}}-\frac{\Theta_{\beta_{n+1}}^{k} x_{n+1}-\Theta_{\beta_{n+1}}^{k-1} x_{n+1}}{\beta_{n+1}}\right\rangle \geq 0
$$

and hence

$$
\begin{aligned}
& \left\|\Theta_{\beta_{n+1}}^{k} x_{n+1}-\Theta_{\beta_{n}}^{k} x_{n}\right\|^{2} \\
& \quad \leq\left\langle\Theta_{\beta_{n+1}}^{k} x_{n+1}-\Theta_{\beta_{n}}^{k} x_{n},\left(\Theta_{\beta_{n+1}}^{k-1} x_{n+1}-\Theta_{\beta_{n}}^{k-1} x_{n}\right)+\left(1-\frac{\beta_{n}}{\beta_{n+1}}\right)\left(\Theta_{\beta_{n+1}}^{k} x_{n+1}-\Theta_{\beta_{n+1}}^{k-1} x_{n+1}\right)\right\rangle .
\end{aligned}
$$

Without loss of generality, let us assume that there exists a real number $d$ such that $\beta_{n}>d>0$ for all $n=1,2, \ldots$. Hence, for each $k=1,2, \ldots, m$ we have

$$
\begin{aligned}
& \left\|\Theta_{\beta_{n+1}}^{k} x_{n+1}-\Theta_{\beta_{n}}^{k} x_{n}\right\| \leq\left\|\Theta_{\beta_{n+1}}^{k-1} x_{n+1}-\Theta_{\beta_{n}}^{k-1} x_{n}\right\|+\frac{1}{\beta_{n+1}}\left|\beta_{n+1}-\beta_{n}\right|\left\|\Theta_{\beta_{n+1}}^{k} x_{n+1}-\Theta_{\beta_{n+1}}^{k-1} x_{n+1}\right\| \\
& \leq\left\|\Theta_{\beta_{n+1}}^{k-1} x_{n+1}-\Theta_{\beta_{n}}^{k-1} x_{n}\right\|+\frac{1}{d}\left|\beta_{n+1}-\beta_{n}\right| M_{0},
\end{aligned}
$$


where $M_{0}$ is an approximate constant such that

$$
M_{0} \geq \max \left\{\sup _{n \geq 1}\left\{\left\|\Theta_{\beta_{n+1}}^{k} x_{n+1}-\Theta_{\beta_{n+1}}^{k-1} x_{n+1}\right\|\right\}, k=1,2, \ldots, m\right\} .
$$

It follows from (3.14) that

$$
\left\|u_{n+1}-u_{n}\right\|=\left\|\Theta_{\beta_{n+1}^{m}}^{m} x_{n+1}-\Theta_{\beta_{n}}^{m} x_{n}\right\| \leq\left\|x_{n+1}-x_{n}\right\|+\frac{m}{d}\left|\beta_{n+1}-\beta_{n}\right| M_{0} .
$$

Put $\rho_{n}=P_{C}\left(I-r_{n} A\right) y_{n}$. We have

$$
\begin{aligned}
\left\|y_{n}-y_{n+1}\right\| & =\left\|P_{C}\left(I-s_{n} A\right) u_{n}-P_{C}\left(I-s_{n+1} A\right) u_{n+1}\right\| \\
& \leq\left\|\left(I-s_{n} A\right) u_{n}-\left(I-s_{n+1} A\right) u_{n+1}\right\| \\
& =\left\|\left(u_{n}-s_{n} A u_{n}\right)-\left(u_{n+1}-s_{n} A u_{n+1}\right)+\left(s_{n+1}-s_{n}\right) A u_{n+1}\right\| \\
& \leq\left\|u_{n}-u_{n+1}\right\|+\left|s_{n+1}-s_{n}\right| M_{1},
\end{aligned}
$$

where $M_{1}$ is an approximate constant such that $M_{1} \geq \max \left\{\sup _{n \geq 1}\left\{\left\|A u_{n}\right\|\right\}, M_{0}\right\}$.

Substituting (3.16) into (3.17), we have

$$
\left\|y_{n}-y_{n+1}\right\| \leq\left\|x_{n+1}-x_{n}\right\|+\left[\frac{m}{d}\left|\beta_{n+1}-\beta_{n}\right|+\left|s_{n+1}-s_{n}\right|\right] M_{1} .
$$

It follows from (3.18) that

$$
\begin{aligned}
\left\|\rho_{n}-\rho_{n+1}\right\| & =\left\|P_{C}\left(I-r_{n} A\right) y_{n}-P_{C}\left(I-r_{n+1} A\right) y_{n+1}\right\| \\
& \leq\left\|\left(I-r_{n} A\right) y_{n}-\left(I-r_{n+1} A\right) y_{n+1}\right\| \\
& =\left\|\left(y_{n}-r_{n} A y_{n}\right)-\left(y_{n+1}-r_{n} A y_{n+1}\right)+\left(r_{n+1}-r_{n}\right) A y_{n+1}\right\| \\
& \leq\left\|y_{n}-y_{n+1}\right\|+\left|r_{n+1}-r_{n}\right| M_{2} \\
& \leq\left\|x_{n}-x_{n+1}\right\|+\left[\frac{m}{d}\left|\beta_{n+1}-\beta_{n}\right|+\left|s_{n+1}-s_{n}\right|+\left|r_{n+1}-r_{n}\right|\right] M_{2},
\end{aligned}
$$

where $M_{2}$ is an approximate constant such that $M_{2} \geq \max \left\{M_{1}, \sup _{n \geq 1}\left\{\left\|A y_{n+1}\right\|\right\}\right\}$.

Observe that

$$
\begin{gathered}
x_{n+1}=\alpha_{n} \gamma f\left(W_{n} x_{n}\right)+\left(I-\alpha_{n} B\right) W_{n} \rho_{n}, \\
x_{n+2}=\alpha_{n+1} \gamma f\left(W_{n+1} x_{n+1}\right)+\left(I-\alpha_{n+1} B\right) W_{n+1} \rho_{n+1},
\end{gathered}
$$

we have

$$
\begin{aligned}
x_{n+2}-x_{n+1}= & \alpha_{n+1} \gamma\left[f\left(W_{n+1} x_{n+1}\right)-f\left(W_{n} x_{n}\right)\right]+\left(I-\alpha_{n+1} B\right)\left(W_{n+1} \rho_{n+1}-W_{n} \rho_{n}\right) \\
& +\left(\alpha_{n+1}-\alpha_{n}\right)\left[\gamma f\left(W_{n} x_{n}\right)-B W_{n} \rho_{n}\right] .
\end{aligned}
$$


It follows that

$$
\begin{aligned}
\left\|x_{n+2}-x_{n+1}\right\| \leq & \alpha_{n+1} \gamma \alpha\left\|W_{n+1} x_{n+1}-W_{n} x_{n}\right\|+\left(1-\alpha_{n+1} \tilde{\gamma}\right)\left\|W_{n+1} \rho_{n+1}-W_{n} \rho_{n}\right\| \\
& +\left|\alpha_{n+1}-\alpha_{n}\right|\left\|\gamma f\left(W_{n} x_{n}\right)-B W_{n} \rho_{n}\right\| \\
\leq & \alpha_{n+1} \gamma \alpha\left(\left\|x_{n+1}-x_{n}\right\|+\left\|W_{n+1} x_{n}-W_{n} x_{n}\right\|\right) \\
& +\left(1-\alpha_{n+1} \tilde{\gamma}\right)\left(\left\|\rho_{n+1}-\rho_{n}\right\|+\left\|W_{n+1} \rho_{n}-W_{n} \rho_{n}\right\|\right) \\
& +\left|\alpha_{n+1}-\alpha_{n}\right|\left\|\gamma f\left(W_{n} x_{n}\right)-B W_{n} \rho_{n}\right\| .
\end{aligned}
$$

Next we estimate $\left\|W_{n+1} x_{n}-W_{n} x_{n}\right\|$ and $\left\|W_{n+1} \rho_{n}-W_{n} \rho_{n}\right\|$. It follows from the definition of $W_{n}$ and nonexpansiveness of $S_{i}$ that

$$
\begin{aligned}
\left\|W_{n+1} x_{n}-W_{n} x_{n}\right\| & =\left\|U_{n+1,1} x_{n}-U_{n, 1} x_{n}\right\| \\
& =\left\|\xi_{1} S_{1} U_{n+1,2} x_{n}+\left(1-\xi_{1}\right) x_{n}-\left\{\xi_{1} S_{1} U_{n, 2} x_{n}+\left(1-\xi_{1}\right) x_{n}\right\}\right\| \\
& =\xi_{1}\left\|S_{1} U_{n+1,2} x_{n}-S_{1} U_{n, 2} x_{n}\right\| \\
& \leq \xi_{1}\left\|U_{n+1,2} x_{n}-U_{n, 2} x_{n}\right\| \\
& =\xi_{1}\left\|\xi_{2} S_{2} U_{n+1,3} x_{n}+\left(1-\xi_{2}\right) x_{n}-\left\{\xi_{2} S_{2} U_{n, 3} x_{n}+\left(1-\xi_{2}\right) x_{n}\right\}\right\| \\
& =\xi_{1} \xi_{2}\left\|S_{2} U_{n+1,3} x_{n}-S_{2} U_{n, 3} x_{n}\right\| \\
& \leq \xi_{1} \xi_{2}\left\|U_{n+1,3} x_{n}-U_{n, 3} x_{n}\right\| \\
& \vdots \\
& \leq \prod_{i=1}^{n} \xi_{i}\left\|U_{n+1, n+1} x_{n}-U_{n, n+1} x_{n}\right\| \\
& =\prod_{i=1}^{n} \xi_{i}\left\|\xi_{n+1} S_{n+1} x_{n}+\left(1-\xi_{n+1}\right) x_{n}-x_{n}\right\| \\
& =\prod_{i=1}^{n+1} \xi_{i}\left\|S_{n+1} x_{n}-x_{n}\right\| \\
& \leq \prod_{i=1}^{n+1} \xi_{i} M_{3}
\end{aligned}
$$

where $M_{3}$ is an approximate constant such that

$$
M_{3} \geq \max \left\{M_{2}, \sup _{n \geq 1}\left\{\left\|S_{n+1} x_{n}-x_{n}\right\|\right\}, \sup _{n \geq 1}\left\{\left\|S_{n+1} \rho_{n}-\rho_{n}\right\|\right\}\right\} .
$$

Similarly, we have

$$
\left\|W_{n+1} \rho_{n}-W_{n} \rho_{n}\right\| \leq \Pi_{i=1}^{n+1} \xi_{i} M_{3}
$$


Substituting (3.19), (3.23), and (3.25) into (3.22) yields that

$$
\begin{aligned}
\| x_{n+2} & -x_{n+1} \| \\
\leq & \alpha_{n+1} \gamma \alpha\left(\left\|x_{n+1}-x_{n}\right\|+\prod_{i=1}^{n+1} \xi_{i} M_{3}\right) \\
& +\left(1-\alpha_{n+1} \tilde{\gamma}\right)\left(\left\|x_{n+1}-x_{n}\right\|+\left[\frac{m}{d}\left|\beta_{n+1}-\beta_{n}\right|+\left|s_{n+1}-s_{n}\right|+\left|r_{n+1}-r_{n}\right|\right] M_{2}+\Pi_{i=1}^{n+1} \xi_{i} M_{3}\right) \\
& +\left|\alpha_{n+1}-\alpha_{n}\right|\left\|\gamma f\left(W_{n} x_{n}\right)-B W_{n} \rho_{n}\right\| \\
\leq & {\left[1-\alpha_{n+1}(\tilde{\gamma}-\gamma \alpha)\right]\left\|x_{n+1}-x_{n}\right\|+M_{4}\left(\frac{m}{d}\left|\beta_{n+1}-\beta_{n}\right|+\left|s_{n+1}-s_{n}\right|+\left|r_{n+1}-r_{n}\right|+\left|\alpha_{n+1}-\alpha_{n}\right|\right), } \\
& +\prod_{i=1}^{n+1} \xi_{i}
\end{aligned}
$$

where $M_{4}$ is an approximate constant such that

$$
M_{4} \geq \max \left\{M_{3}, \sup _{n \geq 1}\left\{\left\|\gamma f\left(W_{n} x_{n}\right)-B W_{n} \rho_{n}\right\|\right\}\right\} .
$$

It follows from conditions $(\mathrm{C} 1)-(\mathrm{C} 3)$ and $\Pi_{i=1}^{n+1} \xi_{i} \leq \delta^{n+1}$ and Lemma 2.2 that

$$
\left\|x_{n+1}-x_{n}\right\| \longrightarrow 0
$$

Observe that

$$
x_{n+1}-W_{n} \rho_{n}=\alpha_{n}\left(\gamma f\left(W_{n} x_{n}\right)-B W_{n} \rho_{n}\right),
$$

it follows from (C1) that

$$
\lim _{n \rightarrow \infty}\left\|W_{n} \rho_{n}-x_{n+1}\right\|=0 .
$$

For $p \in \Omega$, we have

$$
\begin{aligned}
\left\|y_{n}-p\right\|^{2} & =\left\|P_{C}\left(I-s_{n} A\right) u_{n}-P_{C}\left(I-s_{n} A\right) p\right\|^{2} \\
& \leq\left\|\left(u_{n}-p\right)-s_{n}\left(A u_{n}-A p\right)\right\|^{2} \\
& =\left\|u_{n}-p\right\|^{2}-2 s_{n}\left\langle u_{n}-p, A u_{n}-A p\right\rangle+s_{n}^{2}\left\|A u_{n}-A p\right\|^{2} \\
& \leq\left\|x_{n}-p\right\|^{2}-2 s_{n}\left[-u\left\|A u_{n}-A p\right\|^{2}+v\left\|u_{n}-p\right\|^{2}\right]+s_{n}^{2}\left\|A u_{n}-A p\right\|^{2} \\
& \leq\left\|x_{n}-p\right\|^{2}+\left(2 s_{n} u+s_{n}^{2}-\frac{2 s_{n} v}{\mu^{2}}\right)\left\|A u_{n}-A p\right\|^{2} .
\end{aligned}
$$


Similarly, we have

$$
\left\|\rho_{n}-p\right\|^{2} \leq\left\|x_{n}-p\right\|^{2}+\left(2 r_{n} u+r_{n}^{2}-\frac{2 r_{n} v}{\mu^{2}}\right)\left\|A y_{n}-A p\right\|^{2}
$$

On the other hand, we have

$$
\begin{aligned}
\left\|x_{n+1}-p\right\|^{2} & =\left\|\alpha_{n}\left(\gamma f\left(W_{n} x_{n}\right)-B p\right)+\left(I-\alpha_{n} B\right)\left(W_{n} \rho_{n}-p\right)\right\|^{2} \\
& \leq\left(\alpha_{n}\left\|\gamma f\left(W_{n} x_{n}\right)-B p\right\|+\left(1-\alpha_{n} \tilde{\gamma}\right)\left\|\rho_{n}-p\right\|\right)^{2} \\
& \leq \alpha_{n}\left\|\gamma f\left(W_{n} x_{n}\right)-B p\right\|^{2}+\left\|\rho_{n}-p\right\|^{2}+2 \alpha_{n}\left\|\rho_{n}-p\right\|\left\|\gamma f\left(W_{n} x_{n}\right)-B p\right\| .
\end{aligned}
$$

Substituting (3.32) into (3.33), we have

$$
\begin{aligned}
\left\|x_{n+1}-p\right\|^{2} \leq & \alpha_{n}\left\|\gamma f\left(W_{n} x_{n}\right)-B p\right\|^{2}+\left\|x_{n}-p\right\|^{2}+\left(2 r_{n} u+r_{n}^{2}-\frac{2 r_{n} v}{\mu^{2}}\right)\left\|A y_{n}-A p\right\|^{2} \\
& +2 \alpha_{n}\left\|\rho_{n}-p\right\|\left\|\gamma f\left(W_{n} x_{n}\right)-B p\right\| .
\end{aligned}
$$

It follows from condition (C2) that

$$
\begin{aligned}
&\left(\frac{2 a v}{\mu^{2}}-2 b u-b^{2}\right)\left\|A y_{n}-A p\right\|^{2} \\
& \leq \alpha_{n}\left\|\gamma f\left(W_{n} x_{n}\right)-B p\right\|^{2}+\left\|x_{n}-p\right\|^{2} \\
&-\left\|x_{n+1}-p\right\|^{2}+2 \alpha_{n}\left\|\rho_{n}-p\right\|\left\|\gamma f\left(W_{n} x_{n}\right)-B p\right\| \\
& \leq \alpha_{n}\left\|\gamma f\left(W_{n} x_{n}\right)-B p\right\|^{2}+\left(\left\|x_{n}-p\right\|+\left\|x_{n+1}-p\right\|\right)\left\|x_{n+1}-x_{n}\right\| \\
&+2 \alpha_{n}\left\|\rho_{n}-p\right\|\left\|\gamma f\left(W_{n} x_{n}\right)-B p\right\| .
\end{aligned}
$$

As $\left\|x_{n+1}-x_{n}\right\| \rightarrow 0$ and $\lim _{n \rightarrow \infty} \alpha_{n}=0$, we have

$$
\lim _{n \rightarrow \infty}\left\|A y_{n}-A p\right\|=0
$$

It is easy to see that $\left\|\rho_{n}-p\right\| \leq\left\|y_{n}-p\right\|$. Using (3.33) again, we have

$$
\left\|x_{n+1}-p\right\|^{2} \leq \alpha_{n}\left\|\gamma f\left(W_{n} x_{n}\right)-B p\right\|^{2}+\left\|y_{n}-p\right\|^{2}+2 \alpha_{n}\left\|\rho_{n}-p\right\|\left\|\gamma f\left(W_{n} x_{n}\right)-B p\right\| .
$$

Substituting (3.31) into (3.37), we can obtain

$$
\begin{aligned}
\left\|x_{n+1}-p\right\|^{2} \leq & \alpha_{n}\left\|\gamma f\left(W_{n} x_{n}\right)-B p\right\|^{2}+\left\|x_{n}-p\right\|^{2}+\left(2 s_{n} u+s_{n}^{2}-\frac{2 s_{n} v}{\mu^{2}}\right)\left\|A u_{n}-A p\right\|^{2} \\
& +2 \alpha_{n}\left\|\rho_{n}-p\right\|\left\|\gamma f\left(W_{n} x_{n}\right)-B p\right\| .
\end{aligned}
$$


It follows from (C2) that

$$
\begin{aligned}
\left(\frac{2 a v}{\mu^{2}}-2 b v-b^{2}\right)\left\|A u_{n}-A p\right\|^{2} & \\
\leq & \alpha_{n}\left\|r f\left(W_{n} x_{n}\right)-B p\right\|^{2}+\left\|x_{n}-p\right\|^{2}-\left\|x_{n+1}-p\right\|^{2} \\
& +2 \alpha_{n}\left\|\rho_{n}-p\right\|\left\|\gamma f\left(W_{n} x_{n}\right)-B p\right\| \\
\leq & \alpha_{n}\left\|\gamma f\left(W_{n} x_{n}\right)-B p\right\|^{2}+\left(\left\|x_{n}-p\right\|-\left\|x_{n+1}-p\right\|\right)\left\|x_{n+1}-x_{n}\right\| \\
& +2 \alpha_{n}\left\|\rho_{n}-p\right\|\left\|\gamma f\left(W_{n} x_{n}\right)-B p\right\| .
\end{aligned}
$$

As $\left\|x_{n+1}-x_{n}\right\| \rightarrow 0$ and $\lim _{n \rightarrow \infty} \alpha_{n}=0$, we have

$$
\lim _{n \rightarrow \infty}\left\|A u_{n}-A p\right\|=0 \text {. }
$$

Observe that

$$
\begin{aligned}
\left\|\rho_{n}-p\right\|^{2}= & \left\|P_{C}\left(I-r_{n} A\right) y_{n}-P_{C}\left(I-r_{n} A\right) p\right\|^{2} \\
\leq & \left\langle\left(I-r_{n} A\right) y_{n}-\left(I-r_{n} A\right) p, \rho_{n}-p\right\rangle \\
= & \frac{1}{2}\left\{\left\|\left(I-r_{n} A\right) y_{n}-\left(I-r_{n} A\right) p\right\|^{2}+\left\|\rho_{n}-p\right\|^{2}\right. \\
& \left.\quad-\left\|\left(I-r_{n} A\right) y_{n}-\left(I-r_{n} A\right) p-\left(\rho_{n}-p\right)\right\|^{2}\right\} \\
\leq & \frac{1}{2}\left\{\left\|y_{n}-p\right\|^{2}+\left\|\rho_{n}-p\right\|^{2}-\left\|\left(y_{n}-\rho_{n}\right)-r_{n}\left(A y_{n}-A p\right)\right\|^{2}\right\} \\
\leq & \frac{1}{2}\left\{\left\|x_{n}-p\right\|^{2}+\left\|\rho_{n}-p\right\|^{2}-\left\|y_{n}-\rho_{n}\right\|^{2}-r_{n}^{2}\left\|A y_{n}-A p\right\|^{2}\right. \\
\quad & \left.+2 r_{n}\left\langle y_{n}-\rho_{n}, A y_{n}-A p\right\rangle\right\},
\end{aligned}
$$

which yields that

$$
\left\|\rho_{n}-p\right\|^{2} \leq\left\|x_{n}-p\right\|^{2}-\left\|y_{n}-\rho_{n}\right\|^{2}+2 r_{n}\left\|y_{n}-\rho_{n}\right\|\left\|A y_{n}-A p\right\| .
$$

Substituting (3.42) into (3.33) we have

$$
\begin{aligned}
\left\|x_{n+1}-p\right\|^{2} \leq & \alpha_{n}\left\|\gamma f\left(W_{n} x_{n}\right)-B p\right\|^{2}+\left\|x_{n}-p\right\|^{2}-\left\|y_{n}-\rho_{n}\right\|^{2} \\
& +2 r_{n}\left\|y_{n}-\rho_{n}\right\|\left\|A y_{n}-A p\right\|+2 \alpha_{n}\left\|\rho_{n}-p\right\|\left\|\gamma f\left(W_{n} x_{n}\right)-B p\right\|,
\end{aligned}
$$


which implies that

$$
\begin{aligned}
\left\|y_{n}-\rho_{n}\right\|^{2} \leq & \alpha_{n}\left\|\gamma f\left(W_{n} x_{n}\right)-B p\right\|^{2}+\left\|x_{n}-p\right\|^{2}-\left\|x_{n+1}-p\right\|^{2} \\
& +2 r_{n}\left\|y_{n}-\rho_{n}\right\|\left\|A y_{n}-A p\right\|+2 \alpha_{n}\left\|\rho_{n}-p\right\|\left\|\gamma f\left(W_{n} x_{n}\right)-B p\right\| \\
\leq & \alpha_{n}\left\|\gamma f\left(W_{n} x_{n}\right)-B p\right\|^{2}+\left(\left\|x_{n}-p\right\|+\left\|x_{n+1}-p\right\|\right)\left\|x_{n+1}-x_{n}\right\| \\
& +2 r_{n}\left\|y_{n}-\rho_{n}\right\|\left\|A y_{n}-A p\right\|+2 \alpha_{n}\left\|\rho_{n}-p\right\|\left\|\gamma f\left(W_{n} x_{n}\right)-B p\right\| .
\end{aligned}
$$

It follows from (C1), $\left\|x_{n+1}-x_{n}\right\| \rightarrow 0$, and $\left\|A y_{n}-A p\right\| \rightarrow 0$ that $\lim _{n \rightarrow \infty}\left\|y_{n}-\rho_{n}\right\|=0$.

For $p \in \Omega$, we have

$$
\begin{aligned}
& \left\|y_{n}-p\right\|^{2} \\
& =\left\|P_{C}\left(I-s_{n} A\right) u_{n}-P_{C}\left(I-s_{n} A\right) p\right\|^{2} \\
& \leq\left\langle P_{C}\left(I-s_{n} A\right) u_{n}-P_{C}\left(I-s_{n} A\right) p,\left(I-s_{n} A\right) u_{n}-\left(I-s_{n} A\right) p\right\rangle \\
& =\left\langle y_{n}-p,\left(I-s_{n} A\right) u_{n}-\left(I-s_{n} A\right) p\right\rangle \\
& =\frac{1}{2}\left(\left\|y_{n}-p\right\|^{2}+\left\|\left(I-s_{n} A\right) u_{n}-\left(I-s_{n} A\right) p\right\|^{2}-\left\|\left(y_{n}-p\right)-\left[u_{n}-p-s_{n}\left(A u_{n}-A p\right)\right]\right\|^{2}\right) \\
& \leq \frac{1}{2}\left(\left\|y_{n}-p\right\|^{2}+\left\|u_{n}-p\right\|^{2}-\left\|y_{n}-p-\left[u_{n}-p-s_{n}\left(A u_{n}-A p\right)\right]\right\|^{2}\right) \\
& =\frac{1}{2}\left(\left\|y_{n}-p\right\|^{2}+\left\|u_{n}-p\right\|^{2}-\left\|y_{n}-u_{n}\right\|^{2}+2 s_{n}\left\langle y_{n}-u_{n}, A u_{n}-A p\right\rangle-s_{n}^{2}\left\|A u_{n}-A p\right\|^{2}\right) .
\end{aligned}
$$

This implies that

$$
\begin{aligned}
\left\|y_{n}-p\right\|^{2} & \leq\left\|u_{n}-p\right\|^{2}-\left\|y_{n}-u_{n}\right\|^{2}+2 s_{n}\left\langle y_{n}-u_{n}, A u_{n}-A p\right\rangle-s_{n}^{2}\left\|A u_{n}-A p\right\|^{2} \\
& \leq\left\|u_{n}-p\right\|^{2}-\left\|y_{n}-u_{n}\right\|^{2}+2 s_{n}\left\|y_{n}-u_{n}\right\|\left\|A u_{n}-A p\right\| .
\end{aligned}
$$

By (3.46), (3.37), and (3.5), we obtain

$$
\begin{aligned}
\left\|x_{n+1}-p\right\|^{2} \leq & \alpha_{n}\left\|\gamma f\left(W_{n} x_{n}\right)-B p\right\|^{2}+\left\|u_{n}-p\right\|^{2}-\left\|y_{n}-u_{n}\right\|^{2}+2 s_{n}\left\|y_{n}-u_{n}\right\|\left\|A u_{n}-A p\right\| \\
& +2 \alpha_{n}\left\|\rho_{n}-p\right\|\left\|\gamma f\left(W_{n} x_{n}\right)-B p\right\| \\
\leq & \alpha_{n}\left\|\gamma f\left(W_{n} x_{n}\right)-B p\right\|^{2}+\left\|x_{n}-p\right\|^{2}-\left\|y_{n}-u_{n}\right\|^{2} \\
& +2 s_{n}\left\|y_{n}-u_{n}\right\|\left\|A u_{n}-A p\right\|+2 \alpha_{n}\left\|\rho_{n}-p\right\|\left\|\gamma f\left(W_{n} x_{n}\right)-B p\right\| .
\end{aligned}
$$


It follows that

$$
\begin{aligned}
\left\|y_{n}-u_{n}\right\|^{2} \leq & \alpha_{n}\left\|\gamma f\left(W_{n} x_{n}\right)-B p\right\|^{2}+\left\|x_{n}-p\right\|^{2}-\left\|x_{n+1}-p\right\|^{2}+2 s_{n}\left\|y_{n}-u_{n}\right\|\left\|A u_{n}-A p\right\| \\
& +2 \alpha_{n}\left\|\rho_{n}-p\right\|\left\|\gamma f\left(W_{n} x_{n}\right)-B p\right\| \\
\leq & \alpha_{n}\left\|\gamma f\left(W_{n} x_{n}\right)-B p\right\|^{2}+\left(\left\|x_{n}-p\right\|+\left\|x_{n+1}-p\right\|\right)\left(\left\|x_{n}-x_{n+1}\right\|\right) \\
& +2 s_{n}\left\|y_{n}-u_{n}\right\|\left\|A u_{n}-A p\right\|+2 \alpha_{n}\left\|\rho_{n}-p\right\|\left\|\gamma f\left(W_{n} x_{n}\right)-B p\right\| .
\end{aligned}
$$

It follows from (C1), $\left\|A u_{n}-A p\right\| \rightarrow 0$, and $\left\|x_{n+1}-x_{n}\right\| \rightarrow 0$ that $\left\|y_{n}-u_{n}\right\| \rightarrow 0$. It follows from $\left\|\rho_{n}-u_{n}\right\| \leq\left\|\rho_{n}-y_{n}\right\|+\left\|y_{n}-u_{n}\right\|$ that $\lim _{n \rightarrow \infty}\left\|u_{n}-\rho_{n}\right\|=0$.

We now show that

$$
\lim _{n \rightarrow \infty}\left\|\Theta_{\beta_{n}}^{k} x_{n}-\Theta_{\beta_{n}}^{k-1} x_{n}\right\|=0, \quad k=1,2, \ldots, m .
$$

Indeed, let $p \in \Omega$, it follows from the firmly nonexpansiveness of $T_{\beta_{n}}^{F_{k}}$, we have for each $k \in\{1,2, \ldots, m\}$,

$$
\begin{aligned}
\left\|\Theta_{\beta_{n}}^{k} x_{n}-p\right\|^{2} & =\left\|T_{\beta_{n}}^{F_{k}} \Theta_{\beta_{n}}^{k-1} x_{n}-T_{\beta_{n}}^{F_{k}} p\right\|^{2} \leq\left\langle\Theta_{\beta_{n}}^{k} x_{n}-p, \Theta_{\beta_{n}}^{k-1} x_{n}-p\right\rangle \\
& =\frac{1}{2}\left(\left\|\Theta_{\beta_{n}}^{k} x_{n}-p\right\|^{2}+\left\|\Theta_{\beta_{n}}^{k-1} x_{n}-p\right\|^{2}-\left\|\Theta_{\beta_{n}}^{k} x_{n}-\Theta_{\beta_{n}}^{k-1} x_{n}\right\|^{2}\right) .
\end{aligned}
$$

Thus, we get

$$
\left\|\Theta_{\beta_{n}}^{k} x_{n}-p\right\|^{2} \leq\left\|\Theta_{\beta_{n}}^{k-1} x_{n}-p\right\|^{2}-\left\|\Theta_{\beta_{n}}^{k} x_{n}-\Theta_{\beta_{n}}^{k-1} x_{n}\right\|^{2}, \quad k=1,2, \ldots, m .
$$

This implies that for each $k \in\{1,2, \ldots, m\}$,

$$
\begin{aligned}
\left\|\Theta_{\beta_{n}}^{k} x_{n}-p\right\|^{2} \leq & \left\|\Theta_{\beta_{n}}^{0} x_{n}-p\right\|^{2}-\left\|\Theta_{\beta_{n}}^{k} x_{n}-\Theta_{\beta_{n}}^{k-1} x_{n}\right\|^{2} \\
& -\left\|\Theta_{\beta_{n}}^{k-1} x_{n}-\Theta_{\beta_{n}}^{k-2} x_{n}\right\|^{2}-\cdots-\left\|\Theta_{\beta_{n}}^{2} x_{n}-\Theta_{\beta_{n}}^{1} x_{n}\right\|^{2}-\left\|\Theta_{\beta_{n}}^{1} x_{n}-\Theta_{\beta_{n}}^{0} x_{n}\right\|^{2} .
\end{aligned}
$$

It follows from $u_{n}=\Theta_{\beta_{n}}^{m} x_{n}$ that for each $k=1,2, \ldots, m$

$$
\left\|u_{n}-p\right\|^{2} \leq\left\|x_{n}-p\right\|^{2}-\left\|\Theta_{\beta_{n}}^{k} x_{n}-\Theta_{\beta_{n}}^{k-1} x_{n}\right\|^{2} .
$$


By (3.37), (3.6), and (3.53), we have that for each $k=1,2, \ldots, m$

$$
\begin{aligned}
\left\|x_{n+1}-p\right\|^{2} \leq & \alpha_{n}\left\|\gamma f\left(W_{n} x_{n}\right)-B p\right\|^{2}+\left\|u_{n}-p\right\|^{2}+2 \alpha_{n}\left\|\rho_{n}-p\right\|\left\|\gamma f\left(W_{n} x_{n}\right)-B p\right\| \\
\leq & \alpha_{n}\left\|\gamma f\left(W_{n} x_{n}\right)-B p\right\|^{2}+\left\|x_{n}-p\right\|^{2}-\left\|\Theta_{\beta_{n}}^{k} x_{n}-\Theta_{\beta_{n}}^{k-1} x_{n}\right\|^{2} \\
& +2 \alpha_{n}\left\|\rho_{n}-p\right\|\left\|\gamma f\left(W_{n} x_{n}\right)-B p\right\| .
\end{aligned}
$$

Thus, we have that for each $k=1,2, \ldots, m$

$$
\begin{aligned}
\left\|\Theta_{\beta_{n}}^{k} x_{n}-\Theta_{\beta_{n}}^{k-1} x_{n}\right\|^{2} \leq & \alpha_{n}\left\|\gamma f\left(W_{n} x_{n}\right)-B p\right\|^{2}+\left\|x_{n}-p\right\|^{2}-\left\|x_{n+1}-p\right\|^{2} \\
& +2 \alpha_{n}\left\|\rho_{n}-p\right\|\left\|\gamma f\left(W_{n} x_{n}\right)-B p\right\| \\
\leq & \alpha_{n}\left\|\gamma f\left(W_{n} x_{n}\right)-B p\right\|^{2}+\left(\left\|x_{n}-p\right\|+\left\|x_{n+1}-p\right\|\right)\left\|x_{n}-x_{n+1}\right\| \\
& +2 \alpha_{n}\left\|\rho_{n}-p\right\|\left\|\gamma f\left(W_{n} x_{n}\right)-B p\right\| .
\end{aligned}
$$

It follows from (C1) and $\left\|x_{n+1}-x_{n}\right\| \rightarrow 0$ that for each $k=1,2, \ldots, m$

$$
\left\|\Theta_{\beta_{n}}^{k} x_{n}-\Theta_{\beta_{n}}^{k-1} x_{n}\right\| \longrightarrow 0 .
$$

Since

$$
\begin{aligned}
\left\|W_{n} \rho_{n}-\rho_{n}\right\| \leq & \left\|W_{n} \rho_{n}-x_{n+1}\right\|+\left\|x_{n+1}-x_{n}\right\|+\left\|x_{n}-\Theta_{\beta_{n}}^{1} x_{n}\right\|+\left\|\Theta_{\beta_{n}}^{1} x_{n}-\Theta_{\beta_{n}}^{2} x_{n}\right\| \\
& +\cdots+\left\|\Theta_{\beta_{n}}^{m-1} x_{n}-\Theta_{\beta_{n}}^{m} x_{n}\right\|+\left\|u_{n}-y_{n}\right\|+\left\|y_{n}-\rho_{n}\right\| .
\end{aligned}
$$

It follows from (3.56) that

$$
\lim _{n \rightarrow \infty}\left\|W_{n} \rho_{n}-\rho_{n}\right\|=0
$$

Observe that

$$
\left\|W \rho_{n}-\rho_{n}\right\| \leq\left\|W \rho_{n}-W_{n} \rho_{n}\right\|+\left\|W_{n} \rho_{n}-\rho_{n}\right\| .
$$

It follows from Remark 2.7 that

$$
\lim _{n \rightarrow \infty}\left\|W \rho_{n}-\rho_{n}\right\|=0
$$


We show that $P_{\Omega}(\gamma f+(I-B))$ is a contraction. Indeed, for all $x, y \in H$, we have

$$
\begin{aligned}
\| P_{\Omega} & (\gamma f+(I-B))(x)-P_{\Omega}(\gamma f+(I-B))(y) \| \\
& \leq\|(\gamma f+(I-B))(x)-(\gamma f+(I-B))(y)\| \\
& \leq \gamma\|f(x)-f(y)\|+\|I-B\|\|x-y\| \\
& \leq \gamma \alpha\|x-y\|+(1-\tilde{\gamma})\|x-y\| \\
& =(\gamma \alpha+1-\tilde{\gamma})\|x-y\| .
\end{aligned}
$$

The Banach's Contraction Mapping Principle guarantees that $P_{\Omega}(\gamma f+(I-B))$ has a unique fixed point, say $q \in H$. That is, $q=P_{\Omega}(\gamma f+(I-B))(q)$.

Next, we show that

$$
\limsup _{n \rightarrow \infty}\left\langle r f(q)-B q, x_{n}-q\right\rangle \leq 0
$$

To show that, we choose a subsequence $\left\{x_{n_{i}}\right\}$ of $x_{n}$ such that

$$
\limsup _{n \rightarrow \infty}\left\langle\gamma f(q)-B q, x_{n}-q\right\rangle=\lim _{i \rightarrow \infty}\left\langle\gamma f(q)-B q, x_{n_{i}}-q\right\rangle .
$$

As $\left\{x_{n_{i}}\right\}$ is bounded, we know that there is a subsequence $\left\{x_{n_{i_{j}}}\right\}$ of $\left\{x_{n_{i}}\right\}$ which converges weakly to $p$. We may assume, without loss of generality, that $x_{n_{i}} \rightarrow p$. From $\left\|\Theta_{\beta_{n}}^{k} x_{n}-\Theta_{\beta_{n}}^{k-1} x_{n}\right\| \rightarrow 0$ for each $k=1,2, \ldots, m$, we obtain that $\Theta_{\beta_{n_{i}}}^{k} x_{n_{i}} \rightarrow p$ for $k=1,2, \ldots, m$. From $\left\|u_{n}-\rho_{n}\right\| \rightarrow 0$, we also obtain that $\rho_{n_{i}} \rightarrow p$. Since $\left\{u_{n i}\right\} \subset C$ and $C$ is closed and convex, we obtain $p \in C$.

Now we show that $p \in \Omega$. Indeed, let us first show that $p \in \operatorname{VI}(C, A)$. Put

$$
T w_{1}= \begin{cases}A w_{1}+N_{C} w_{1} & \text { if } w_{1} \in C, \\ \emptyset & \text { if } w_{1} \notin C .\end{cases}
$$

Since $A$ is relaxed $(u, v)$-cocoercive, we have

$$
\langle A x-A y, x-y\rangle \geq(-u)\|A x-A y\|^{2}+v\|x-y\|^{2} \geq\left(v-u \mu^{2}\right)\|x-y\|^{2} \geq 0,
$$

which yields that $A$ is monotone. Thus $T$ is maximal monotone. Let $\left(w_{1}, w_{2}\right) \in G(T)$. Since $w_{2}-A w_{1} \in N_{C} w_{1}$ and $\rho_{n} \in C$, we have

$$
\left\langle w_{1}-\rho_{n}, w_{2}-A w_{1}\right\rangle \geq 0
$$

On the other hand, from $\rho_{n}=P_{C}\left(I-r_{n} A\right) y_{n}$, we have

$$
\left\langle w_{1}-\rho_{n}, \rho_{n}-\left(I-r_{n} A\right) y_{n}\right\rangle \geq 0
$$


and hence

$$
\left\langle w_{1}-\rho_{n}, \frac{\rho_{n}-y_{n}}{r_{n}}+A y_{n}\right\rangle \geq 0
$$

It follows that

$$
\begin{aligned}
\left\langle w_{1}-\rho_{n_{i}}, w_{2}\right\rangle \geq & \left\langle w_{1}-\rho_{n_{i}}, A w_{1}\right\rangle \\
\geq & \left\langle w_{1}-\rho_{n_{i}}, A w_{1}\right\rangle-\left\langle w_{1}-\rho_{n_{i}}, \frac{\rho_{n_{i}}-y_{n_{i}}}{r_{n_{i}}}+A y_{n_{i}}\right\rangle \\
\geq & \left\langle w_{1}-\rho_{n_{i}}, A w_{1}-\frac{\rho_{n_{i}}-y_{n_{i}}}{r_{n_{i}}}-A y_{n_{i}}\right\rangle \\
= & \left\langle w_{1}-\rho_{n_{i}}, A w_{1}-A \rho_{n_{i}}\right\rangle+\left\langle w_{1}-\rho_{n_{i}}, A \rho_{n_{i}}-A y_{n_{i}}\right\rangle \\
& -\left\langle w_{1}-\rho_{n_{i}}, \frac{\rho_{n_{i}}-y_{n_{i}}}{r_{n_{i}}}\right\rangle \\
\geq & \left\langle w_{1}-\rho_{n_{i}}, A \rho_{n_{i}}-A y_{n_{i}}\right\rangle-\left\langle w_{1}-\rho_{n_{i}}, \frac{\rho_{n_{i}}-y_{n_{i}}}{r_{n_{i}}}\right\rangle,
\end{aligned}
$$

which implies that $\left\langle w_{1}-p, w_{2}\right\rangle \geq 0$. We have $p \in T^{-1} 0$ and hence $p \in \operatorname{VI}(C, A)$.

We next show that $p \in \bigcap_{k=1}^{m} \operatorname{EP}\left(F_{k}\right)$. Indeed, by Lemma 2.1, we have that for each $k=1,2, \ldots, m$,

$$
F_{k}\left(\Theta_{\beta_{n}}^{k} x_{n}, y\right)+\frac{1}{\beta_{n}}\left\langle y-\Theta_{\beta_{n}}^{k} x_{n}, \Theta_{\beta_{n}}^{k} x_{n}-\Theta_{\beta_{n}}^{k-1} x_{n}\right\rangle \geq 0, \quad \forall y \in C
$$

It follows from (A2) that

$$
\frac{1}{\beta_{n}}\left\langle y-\Theta_{\beta_{n}}^{k} x_{n}, \Theta_{\beta_{n}}^{k} x_{n}-\Theta_{\beta_{n}}^{k-1} x_{n}\right\rangle \geq F_{k}\left(y, \Theta_{\beta_{n}}^{k} x_{n}\right), \quad \forall y \in C
$$

Hence,

$$
\left\langle y-\Theta_{\beta_{n_{i}}}^{k} x_{n_{i}}, \frac{\Theta_{\beta_{n_{i}}}^{k} x_{n_{i}}-\Theta_{\beta_{n_{i}}}^{k-1} x_{n_{i}}}{\beta_{n_{i}}}\right\rangle \geq F_{k}\left(y, \Theta_{\beta_{n_{i}}}^{k} x_{n_{i}}\right), \quad \forall y \in C .
$$

It follows from (A4), (A5), $\left(\Theta_{\beta_{n_{i}}}^{k} x_{n_{i}}-\Theta_{\beta_{n_{i}}}^{k-1} x_{n_{i}}\right) / \beta_{n_{i}} \rightarrow 0$, and $\Theta_{\beta_{n_{i}}}^{k} x_{n_{i}} \rightarrow p$ that for each $k=1,2, \ldots, m$,

$$
F_{k}(y, p) \leq 0, \quad \forall y \in C
$$


For $t$ with $0<t \leq 1$ and $y \in C$, let $y_{t}=t y+(1-t) p$. Since $y \in C$ and $p \in C$, we obtain $y_{t} \in C$ and hence $F_{k}\left(y_{t}, p\right) \leq 0$. So by (A4), we have

$$
0=F_{k}\left(y_{t}, y_{t}\right) \leq t F_{k}\left(y_{t}, y\right)+(1-t) F_{k}\left(y_{t}, p\right) \leq t F_{k}\left(y_{t}, y\right)
$$

Dividing by $t$, we get that for each $k=1,2, \ldots, m$,

$$
F_{k}\left(y_{t}, y\right) \geq 0
$$

Letting $t \rightarrow 0$, it follows from (A3) that for each $k=1,2, \ldots, m$,

$$
F_{k}(p, y) \geq 0
$$

for all $y \in C$ and hence $p \in \operatorname{EP}\left(F_{k}\right)$ for $k=1,2, \ldots, m$. That is, $p \in \bigcap_{k=1}^{m} \operatorname{EP}\left(F_{k}\right)$.

We now show that $p \in \operatorname{Fix}(W)$. Assume that $p \notin \operatorname{Fix}(W)$. Since $\rho_{n_{i}} \rightarrow p$ and $p \neq W p$, from (3.60) and the Opial condition we have

$$
\begin{aligned}
\liminf _{i \rightarrow \infty}\left\|\rho_{n_{i}}-p\right\| & <\liminf _{i \rightarrow \infty}\left\|\rho_{n_{i}}-W p\right\| \\
& \leq \liminf _{i \rightarrow \infty}\left\{\left\|\rho_{n_{i}}-W \rho_{n_{i}}\right\|+\left\|W \rho_{n_{i}}-W p\right\|\right\} \\
& \leq \liminf _{i \rightarrow \infty}\left\|\rho_{n_{i}}-p\right\|,
\end{aligned}
$$

which is a contradiction. So, we get $p \in \operatorname{Fix}(W)=\bigcap_{i=1}^{\infty} \operatorname{Fix}\left(S_{i}\right)$. This implies that $p \in \Omega$.

Since $q=P_{\Omega}(\gamma f+(I-B))(q)$, we have

$$
\begin{aligned}
\limsup _{n \rightarrow \infty}\left\langle\gamma f(q)-B q, x_{n}-q\right\rangle & =\lim _{i \rightarrow \infty}\left\langle\gamma f(q)-B q, x_{n_{i}}-q\right\rangle \\
& =\langle\gamma f(q)-B q, p-q\rangle \leq 0 .
\end{aligned}
$$

That is, (3.62) holds. Next, we consider

$$
\begin{aligned}
\left\|x_{n+1}-q\right\|^{2}= & \left\|\alpha_{n}\left(\gamma f\left(W_{n} x_{n}\right)-B q\right)+\left(I-\alpha_{n} B\right)\left(W_{n} \rho_{n}-q\right)\right\|^{2} \\
\leq & \left(1-\alpha_{n} \tilde{\gamma}\right)^{2}\left\|W_{n} \rho_{n}-q\right\|^{2}+2 \alpha_{n}\left\langle\gamma f\left(W_{n} x_{n}\right)-B q, x_{n+1}-q\right\rangle \\
\leq & \left(1-\alpha_{n} \tilde{\gamma}\right)^{2}\left\|x_{n}-q\right\|^{2}+2 \alpha_{n} \gamma\left\langle f\left(W_{n} x_{n}\right)-f(q), x_{n+1}-q\right\rangle \\
& +2 \alpha_{n}\left\langle\gamma f(q)-B q, x_{n+1}-q\right\rangle \\
\leq & \left(1-\alpha_{n} \tilde{\gamma}\right)^{2}\left\|x_{n}-q\right\|^{2}+\alpha_{n} \gamma \alpha\left(\left\|x_{n}-q\right\|^{2}+\left\|x_{n+1}-q\right\|^{2}\right) \\
& +2 \alpha_{n}\left\langle\gamma f(q)-B q, x_{n+1}-q\right\rangle
\end{aligned}
$$


So, we can obtain

$$
\begin{aligned}
\left\|x_{n+1}-q\right\|^{2} \leq & \frac{\left(1-\alpha_{n} \tilde{\gamma}\right)^{2}+\alpha_{n} \gamma \alpha}{1-\alpha_{n} \gamma \alpha}\left\|x_{n}-q\right\|^{2}+\frac{2 \alpha_{n}}{1-\alpha_{n} \gamma \alpha}\left\langle\gamma f(q)-B q, x_{n+1}-q\right\rangle \\
= & \frac{1-2 \alpha_{n} \tilde{\gamma}+\alpha_{n} \gamma \alpha}{1-\alpha_{n} \gamma \alpha}\left\|x_{n}-q\right\|^{2}+\frac{\alpha_{n}^{2} \tilde{\gamma}^{2}}{1-\alpha_{n} \gamma \alpha}\left\|x_{n}-q\right\|^{2} \\
& +\frac{2 \alpha_{n}}{1-\alpha_{n} \gamma \alpha}\left\langle\gamma f(q)-B q, x_{n+1}-q\right\rangle \\
\leq & {\left[1-\frac{2 \alpha_{n}(\tilde{\gamma}-\alpha \gamma)}{1-\alpha_{n} \gamma \alpha}\right]\left\|x_{n}-q\right\|^{2} } \\
& +\frac{2 \alpha_{n}(\tilde{\gamma}-\alpha \gamma)}{1-\alpha_{n} \gamma \alpha}\left[\frac{1}{\tilde{\gamma}-\alpha \gamma}\left\langle\gamma f(q)-B q, x_{n+1}-q\right\rangle+\frac{\alpha_{n} \tilde{\gamma}^{2}}{2(\tilde{\gamma}-\alpha \gamma)} M\right],
\end{aligned}
$$

where $M$ is an approximate constant such that $\left.M \geq \sup _{n>1}\left\{\left\|x_{n}-q\right\|^{2}\right\}\right\}$.

Put $l_{n}=2 \alpha_{n}(\tilde{\gamma}-\alpha \gamma) /\left(1-\alpha_{n} \gamma \alpha\right)$ and $t_{n}=(1 /(\tilde{\gamma}-\alpha \gamma))\left\langle\gamma f(q)-B q, x_{n+1}-q\right\rangle+\left(\alpha_{n} \tilde{\gamma}^{2} / 2(\tilde{\gamma}-\right.$ $\alpha \gamma)) M$. That is,

$$
\left\|x_{n+1}-q\right\|^{2} \leq\left(1-l_{n}\right)\left\|x_{n}-q\right\|+l_{n} t_{n}
$$

From condition (C1) and Lemma 2.2, we concluded that $x_{n} \rightarrow q \in \Omega$. It is easy to see that $u_{n} \rightarrow q$ and $y_{n} \rightarrow q$. This completes the proof.

Corollary 3.2. Let $C$ be a nonempty, closed and convex subset of $H$. Let $F$ be a bifunction from $C \times C$ to $\mathbb{R}$ satisfies conditions (A1)-(A5). Let $A: C \rightarrow H$ be relaxed $(u, v)$-cocoercive and $\mu$ Lipschitz continuous and $B$ a strongly positive linear bounded operator on $H$ with coefficient $\tilde{\gamma}>0$. Assume that $0<\gamma<\tilde{\gamma} / \alpha$. Let $S_{1}, S_{2}, \ldots$ be a family of infinitely nonexpansive mappings of $C$ into itself such that $\Gamma=\bigcap_{i=1}^{\infty} \operatorname{Fix}\left(S_{i}\right) \cap V I(C, A) \cap E P(F) \neq \emptyset$, let $\xi_{1}, \xi_{2}, \ldots$ be real numbers such that $0<\xi_{i} \leq \delta<1$ for every $i \in N$ and $W_{n}$ be the $W$-mapping of $C$ into itself generated by $S_{n}, S_{n-1}, \ldots, S_{1}$ and $\xi_{n}, \xi_{n-1}, \ldots, \xi_{1}$. Let $f: C \rightarrow C$ be a contraction with coefficient $\alpha(0<\alpha<1)$ and $\left\{x_{n}\right\},\left\{u_{n}\right\}$ and $\left\{y_{n}\right\}$ be sequences generated by

$$
\begin{gathered}
x_{1}=x \in H, \\
F\left(u_{n}, y\right)+\frac{1}{\beta_{n}}\left\langle y-u_{n}, u_{n}-x_{n}\right\rangle \geq 0, \quad \forall y \in C, \\
y_{n}=P_{C}\left(I-s_{n} A\right) u_{n}, \\
x_{n+1}=\alpha_{n} \gamma f\left(W_{n} x_{n}\right)+\left(I-\alpha_{n} B\right) W_{n} P_{C}\left(I-r_{n} A\right) y_{n}
\end{gathered}
$$


for every $n=1,2, \ldots$, where $\left\{\alpha_{n}\right\},\left\{\beta_{n}\right\},\left\{r_{n}\right\}$ and $\left\{s_{n}\right\}$ are sequences of numbers satisfying the conditions:

(C1) $\left\{\alpha_{n}\right\} \subset[0,1]$ with $\lim _{n \rightarrow \infty} \alpha_{n}=0, \sum_{n=1}^{\infty} \alpha_{n}=\infty$, and $\sum_{n=1}^{\infty}\left|\alpha_{n+1}-\alpha_{n}\right|<\infty$;

(C2) $\left\{r_{n}\right\} \subset[a, b]$ and $\left\{s_{n}\right\} \subset[a, b]$ for some $a, b$ with $0 \leq a \leq b \leq 2\left(v-u \mu^{2}\right) / \mu^{2}, \sum_{n=1}^{\infty} \mid r_{n+1}-$ $r_{n} \mid<\infty$, and $\sum_{n=1}^{\infty}\left|s_{n+1}-s_{n}\right|<\infty$;

(C3) $\liminf _{n \rightarrow \infty} \beta_{n}>0$ and $\sum_{n=1}^{\infty}\left|\beta_{n+1}-\beta_{n}\right|<\infty$. inequality:

Then, $\left\{x_{n}\right\}$ and $\left\{y_{n}\right\}$ converge strongly to $q \in \Gamma$, which solves the following variational

$$
\langle\gamma f q-B q, p-q\rangle \leq 0, \quad \forall p \in \Gamma
$$

Proof. Let $m=1$, by Theorem 3.1, we obtain the desired result.

Corollary 3.3. Let $C$ be a nonempty, closed, and convex subset of $H$. Let $A: C \rightarrow H$ be relaxed $(u, v)$-cocoercive and $\mu$-Lipschitz continuous and let $B$ be a strongly positive linear bounded operator on $H$ with coefficient $\tilde{\gamma}>0$. Assume that $0<\gamma<\tilde{\gamma} / \alpha$. Let $S_{1}, S_{2}, \ldots$ be a family of infinitely nonexpansive mappings of $C$ into itself such that $\Delta=\bigcap_{i=1}^{\infty} \operatorname{Fix}\left(S_{i}\right) \cap V I(C, A) \neq \emptyset$, let $\xi_{1}, \xi_{2}, \ldots$ be real numbers such that $0<\xi_{i} \leq \delta<1$ for every $i \in N$, and let $W_{n}$ be the $W$-mapping of $C$ into itself generated by $S_{n}, S_{n-1}, \ldots, S_{1}$ and $\xi_{n}, \xi_{n-1}, \ldots, \xi_{1}$. Let $f: C \rightarrow C$ be a contraction with coefficient $\alpha(0<\alpha<1)$ and $\left\{x_{n}\right\},\left\{u_{n}\right\}$, and $\left\{y_{n}\right\}$ be sequences generated by

$$
\begin{gathered}
x_{1}=x \in C, \\
y_{n}=P_{C}\left(I-s_{n} A\right) x_{n} \\
x_{n+1}=\alpha_{n} \gamma f\left(W_{n} x_{n}\right)+\left(I-\alpha_{n} B\right) W_{n} P_{C}\left(I-r_{n} A\right) y_{n}
\end{gathered}
$$

for every $n=1,2, \ldots$, where $\left\{\alpha_{n}\right\},\left\{\beta_{n}\right\},\left\{r_{n}\right\}$, and $\left\{s_{n}\right\}$ are sequences of numbers satisfying the conditions:

(C1) $\left\{\alpha_{n}\right\} \subset[0,1]$ with $\lim _{n \rightarrow \infty} \alpha_{n}=0, \sum_{n=1}^{\infty} \alpha_{n}=\infty$, and $\sum_{n=1}^{\infty}\left|\alpha_{n+1}-\alpha_{n}\right|<\infty$;

(C2) $\left\{r_{n}\right\} \subset[a, b]$ and $\left\{s_{n}\right\} \subset[a, b]$ for some $a, b$ with $0 \leq a \leq b \leq 2\left(v-u \mu^{2}\right) / \mu^{2}, \sum_{n=1}^{\infty} \mid r_{n+1}-$ $r_{n} \mid<\infty$ and $\sum_{n=1}^{\infty}\left|s_{n+1}-s_{n}\right|<\infty$. inequality:

Then, $\left\{x_{n}\right\}$ and $\left\{y_{n}\right\}$ converge strongly to $q \in \Delta$, which solves the following variational

$$
\langle\gamma f q-B q, p-q\rangle \leq 0, \quad \forall p \in \Delta
$$

Proof. Let $F(x, y)=0$ for $x, y \in C$, by Corollary 3.2 we obtain the desired result.

Corollary 3.4. Let $C$ be a nonempty, closed and convex subset of $H$. Let $F_{1}, F_{2}, \ldots, F_{m}$ be bifunctions from $C \times C$ to $\mathbb{R}$ satisfies conditions (A1)-(A5). Let $A: C \rightarrow H$ be relaxed $(u, v)$-cocoercive and $\mu$-Lipschitz continuous and B a strongly positive linear bounded operator on $H$ with coefficient $\tilde{\gamma}>0$ 
such that $\Xi=\bigcap_{k=1}^{m} E P\left(F_{k}\right) \cap V I(C, A) \neq \emptyset$. Let $f: C \rightarrow C$ be a contraction with coefficient $\alpha(0<$ $\alpha<1)$ and $\left\{x_{n}\right\},\left\{u_{n}\right\}$ and $\left\{y_{n}\right\}$ be sequences generated by

$$
\begin{gathered}
x_{1}=x \in H, \\
u_{n}=T_{\beta_{n}}^{F_{m}} T_{\beta_{n}}^{F_{m-1}} \cdots T_{\beta_{n}}^{F_{2}} T_{\beta_{n}}^{F_{1}} x_{n}, \\
y_{n}=P_{C}\left(I-s_{n} A\right) u_{n}, \\
x_{n+1}=\alpha_{n} \gamma f\left(x_{n}\right)+\left(I-\alpha_{n} B\right) P_{C}\left(I-r_{n} A\right) y_{n}
\end{gathered}
$$

for every $n=1,2, \ldots$, where $\left\{\alpha_{n}\right\},\left\{\beta_{n}\right\},\left\{r_{n}\right\}$ and $\left\{s_{n}\right\}$ are sequences of numbers satisfying the conditions:

(C1) $\left\{\alpha_{n}\right\} \subset[0,1]$ with $\lim _{n \rightarrow \infty} \alpha_{n}=0, \sum_{n=1}^{\infty} \alpha_{n}=\infty$, and $\sum_{n=1}^{\infty}\left|\alpha_{n+1}-\alpha_{n}\right|<\infty$;

(C2) $\left\{r_{n}\right\} \subset[a, b]$ and $\left\{s_{n}\right\} \subset[a, b]$ for some $a, b$ with $0 \leq a \leq b \leq 2\left(v-u \mu^{2}\right) / \mu^{2}, \sum_{n=1}^{\infty} \mid r_{n+1}-$ $r_{n} \mid<\infty$, and $\sum_{n=1}^{\infty}\left|s_{n+1}-s_{n}\right|<\infty$;

(C3) $\liminf _{n \rightarrow \infty} \beta_{n}>0$ and $\sum_{n=1}^{\infty}\left|\beta_{n+1}-\beta_{n}\right|<\infty$.

Then, $\left\{x_{n}\right\},\left\{y_{n}\right\}$ and $\left\{u_{n}\right\}$ converge strongly to $q \in \Omega$, which solves the following variational inequality:

$$
\langle\gamma f q-B q, p-q\rangle \leq 0, \quad \forall p \in \Xi
$$

Remark 3.5. (i) If $s_{n}=0$ for all $n \geq 0$, by Corollary 3.2, we get Theorem 2.1 in [9]. If $s_{n}=0$ and $S_{i}=I$ for all $n \geq 0$, by Corollary 3.2, we get Theorem 2.1 in [8] with $S=I$. If $s_{n}=0, r_{n}=0$ and $S_{i}=I$ for all $n \geq 0$, by Corollary 3.2, we get Theorem 3.1 in [6] with $S=I$ and Theorem 3.3 in [7] with $S=I$ and $C=H$.

(ii) Corollary 3.3 extends, generalizes and improves the main results in [21, 22, 24].

(iii) It is easy to see that Theorem 3.1 is different from the main results in [1-4].

\section{Acknowledgments}

The authors are grateful to the referee and Proffessor S. Reich for the detailed comments and helpful suggestions which improved the original manuscript greatly. This research was supported by the National Center of Theoretical Sciences (South) of Taiwan, the National Natural Science Foundation of China (Grants 10771228 and 10831009), the Natural Science Foundation of Chongqing (Grant no. CSTC, 2009BB8240), the Research Project of Chongqing Normal University (Grant 08XLZ05), and the project of the Grant NSC 98-2115-M-110-001.

\section{References}

[1] P. L. Combettes and S. A. Hirstoaga, "Equilibrium programming in Hilbert spaces," Journal of Nonlinear and Convex Analysis, vol. 6, pp. 117-136, 2005.

[2] J.-W. Peng and J.-C. Yao, "A viscosity approximation scheme for system of equilibrium problems, nonexpansive mappings and monotone mappings," Nonlinear Analysis: Theory, Methods $\mathcal{E}$ Applications, vol. 71, no. 12, pp. 6001-6010, 2009. 
[3] V. Colao, G. L. Acedo, and G. Marino, "An implicit method for finding common solutions of variational inequalities and systems of equilibrium problems and fixed points of infinite family of nonexpansive mappings," Nonlinear Analysis: Theory, Methods \& Applications, vol. 71, no. 7-8, pp. 27082715, 2009.

[4] S. Saeidi, "Iterative algorithms for finding common solutions of variational inequalities and systems of equilibrium problems and fixed points of families and semigroups of nonexpansive mappings," Nonlinear Analysis: Theory, Methods E Applications, vol. 70, no. 12, pp. 4195-4208, 2009.

[5] S. Takahashi and W. Takahashi, "Viscosity approximation methods for equilibrium problems and fixed point problems in Hilbert spaces," Journal of Mathematical Analysis and Applications, vol. 331, no. 1, pp. 506-515, 2007.

[6] M. Shang, Y. Su, and X. Qin, "A general iterative method for equilibrium problems and fixed point problems in Hilbert spaces," Fixed Point Theory and Applications, vol. 2007, Article ID 95412, 9 pages, 2007.

[7] S. Plubtieng and R. Punpaeng, "A general iterative method for equilibrium problems and fixed point problems in Hilbert spaces," Journal of Mathematical Analysis and Applications, vol. 336, no. 1, pp. 455$469,2007$.

[8] X. Qin, M. Shang, and Y. Su, "A general iterative method for equilibrium problems and fixed point problems in Hilbert spaces," Nonlinear Analysis: Theory, Methods E Applications, vol. 69, no. 11, pp. 3897-3909, 2008.

[9] X. Qin, M. Shang, and Y. Su, "Strong convergence of a general iterative algorithm for equilibrium problems and variational inequality problems," Mathematical and Computer Modelling, vol. 48, no. 7-8, pp. 1033-1046, 2008.

[10] E. Blum and W. Oettli, "From optimization and variational inequalities to equilibrium problems," Mathematics Students, vol. 63, pp. 123-145, 1994.

[11] A. Tada and W. Takahashi, "Weak and strong convergence theorems for a nonexpansive mapping and an equilibrium problem," Journal of Optimization Theory and Applications, vol. 133, no. 3, pp. 359-370, 2007.

[12] S. Plubtieng and R. Punpaeng, "A new iterative method for equilibrium problems and fixed point problems of nonexpansive mappings and monotone mappings," Applied Mathematics and Computation, vol. 197, no. 2, pp. 548-558, 2008.

[13] V. Colao, G. Marino, and H.-K. Xu, "An iterative method for finding common solutions of equilibrium and fixed point problems," Journal of Mathematical Analysis and Applications, vol. 344, no. 1, pp. 340352, 2008.

[14] J.-W. Peng and J.-C. Yao, “A new hybrid-extragradient method for generalized mixed equilibrium problems, fixed point problems and variational inequality problems," Taiwanese Journal of Mathematics, vol. 12, no. 6, pp. 1401-1432, 2008.

[15] J.-W. Peng and J.-C. Yao, "Ishikawa iterative algorithms for a generalized equilibrium problem and fixed point problems of a pseudo-contraction mapping," Journal of Global Optimization, vol. 46, no. 3, pp. 331-345, 2010.

[16] J.-W. Peng and J.-C. Yao, "A modified CQ method for equilibrium problems, fixed points and variational inequality," Fixed Point Theory, vol. 9, no. 2, pp. 515-531, 2008.

[17] S.-S. Chang, H. W. Joseph Lee, and C. K. Chan, "A new method for solving equilibrium problem fixed point problem and variational inequality problem with application to optimization," Nonlinear Analysis: Theory, Methods \& Applications, vol. 70, no. 9, pp. 3307-3319, 2009.

[18] L.-C. Ceng, S. Al-Homidan, Q. H. Ansari, and J.-C. Yao, "An iterative scheme for equilibrium problems and fixed point problems of strict pseudo-contraction mappings," Journal of Computational and Applied Mathematics, vol. 223, no. 2, pp. 967-974, 2009.

[19] A. N. Iusem and W. Sosa, "Iterative algorithms for equilibrium problems," Optimization, vol. 52, no. 3, pp. 301-316, 2003.

[20] T. T. V. Nguyen, J. J. Strodiot, and V. H. Nguyen, "A bundle method for solving equilibrium problems," Mathematical Programming, vol. 116, no. 1-2, pp. 529-552, 2009.

[21] H. K. Xu, "An iterative approach to quadratic optimization," Journal of Optimization Theory and Applications, vol. 116, no. 3, pp. 659-678, 2003.

[22] G. Marino and H.-K. Xu, "A general iterative method for nonexpansive mappings in Hilbert spaces," Journal of Mathematical Analysis and Applications, vol. 318, no. 1, pp. 43-52, 2006.

[23] A. Moudafi, "Viscosity approximation methods for fixed-points problems," Journal of Mathematical Analysis and Applications, vol. 241, no. 1, pp. 46-55, 2000. 
[24] X. Qin, M. Shang, and H. Zhou, "Strong convergence of a general iterative method for variational inequality problems and fixed point problems in Hilbert spaces," Applied Mathematics and Computation, vol. 200, no. 1, pp. 242-253, 2008.

[25] Z. Opial, "Weak convergence of the sequence of successive approximation for nonexpansive mappings," Bulletin of the American Mathematical Society, vol. 73, pp. 561-597, 1967.

[26] R. T. Rockafellar, "On the maximality of sums of nonlinear monotone operators," Transactions of the American Mathematical Society, vol. 149, pp. 75-88, 1970.

[27] H.-K. Xu, "Iterative algorithms for nonlinear operators," Journal of the London Mathematical Society, vol. 66, no. 1, pp. 240-256, 2002.

[28] W. Takahashi and K. Shimoji, "Convergence theorems for nonexpansive mappings and feasibility problems," Mathematical and Computer Modelling, vol. 32, no. 11-13, pp. 1463-1471, 2000.

[29] K. Shimoji and W. Takahashi, "Strong convergence to common fixed points of infinite nonexpansive mappings and applications," Taiwanese Journal of Mathematics, vol. 5, no. 2, pp. 387-404, 2001. 


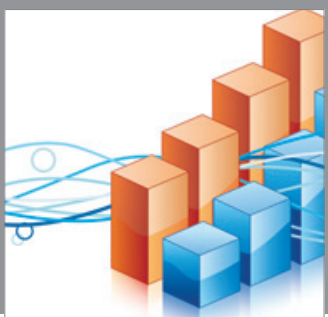

Advances in

Operations Research

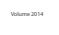

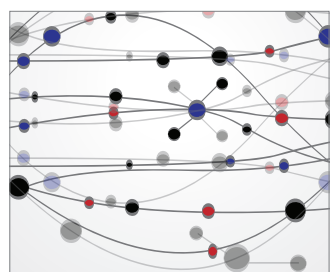

\section{The Scientific} World Journal
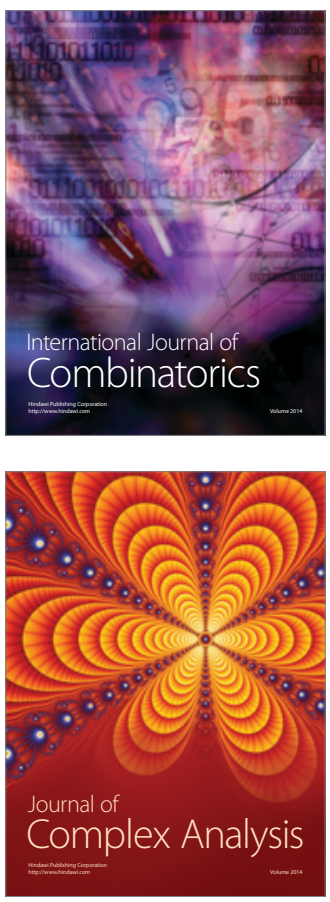

International Journal of

Mathematics and

Mathematical

Sciences
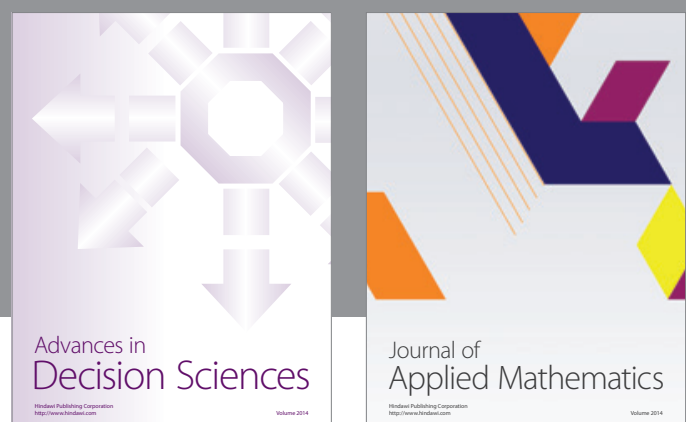

Journal of

Applied Mathematics
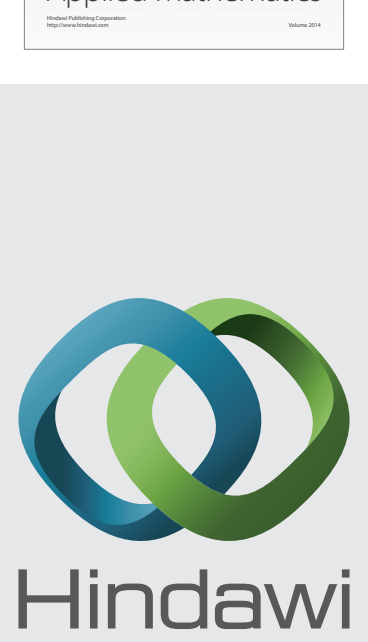

Submit your manuscripts at http://www.hindawi.com
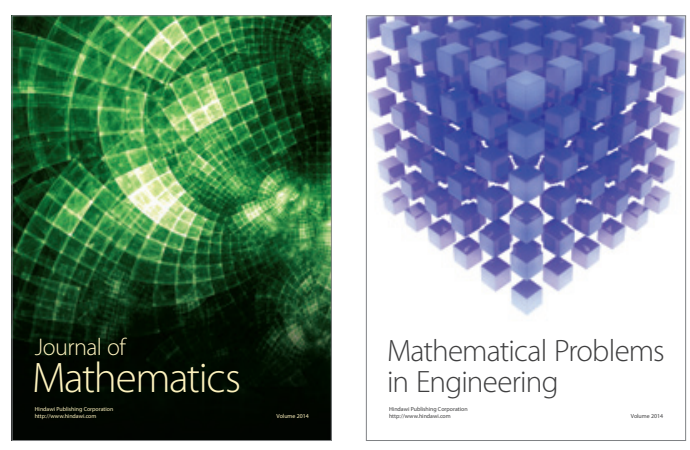

Mathematical Problems in Engineering
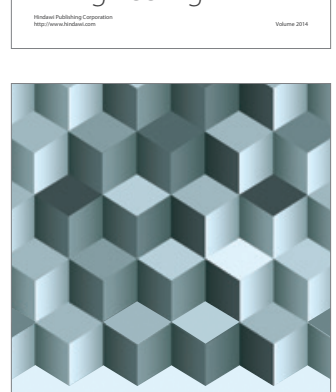

Journal of

Function Spaces
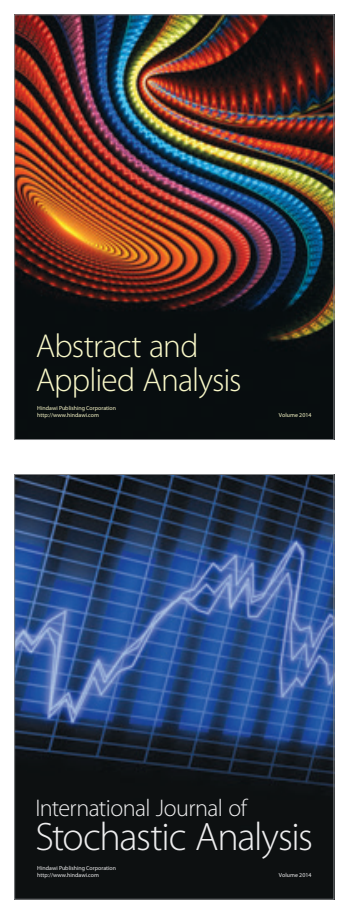

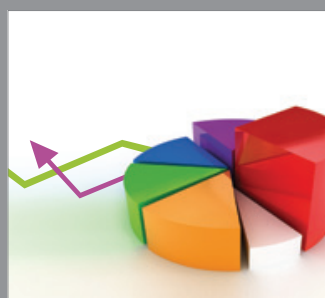

ournal of

Probability and Statistics

Promensencen
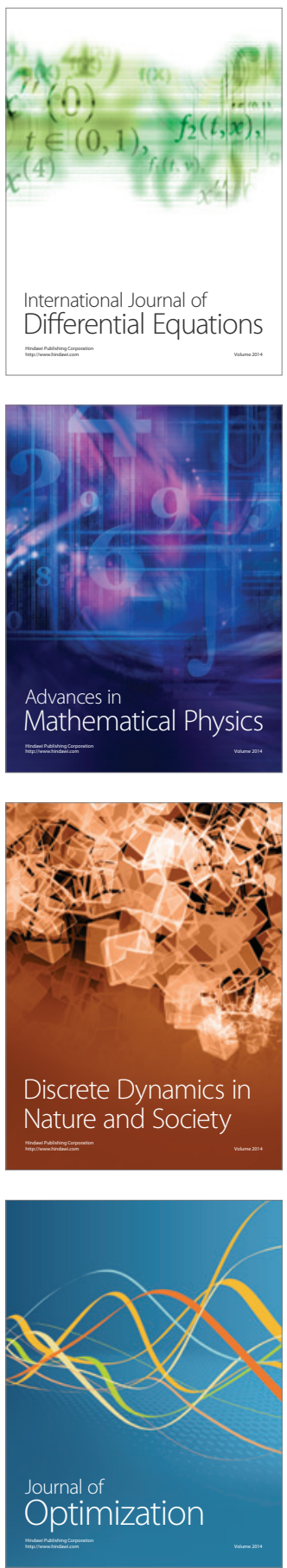